\title{
Kondo Effect in Spin Chains
}

\author{
Nicolas Laflorencie \\ Laboratoire de Physique des Solides, Université Paris-Sud, UMR-8502 CNRS, 91405 \\ Orsay, France \\ Laboratoire de Physique Théorique, IRSAMC, Université Paul Sabatier, CNRS, \\ 31062 Toulouse, France
}

\author{
Erik S. Sørensen $\ddagger$ \\ Department of Physics, University of Toronto, Toronto, ON, M5S 1A7, Canada
}

\author{
Ian Affleck \\ Department of Physics \& Astronomy, University of British Columbia, Vancouver, \\ B.C., Canada, V6T $1 \mathrm{Z1}$ \\ E-mail: laflorencie@lps.u-psud.fr, sorensen@mcmaster.ca, \\ iaffleck@physics.ubc.ca
}

\begin{abstract}
It is well-known that the free electron Kondo problem can be described by a one-dimensional (1D) model because only the s-wave part of the electronic wavefunction is affected by the Kondo coupling. Moreover, since only the spin degrees of freedom are involved in the Kondo interaction, and due to spin-charge separation in 1D, the universal low energy long distance physics of the Kondo model also arises when a magnetic impurity is coupled to the end of a gap-less antiferromagnetic $J_{1}-J_{2}$ spin- $\frac{1}{2}$ chain, where $J_{1}\left(J_{2}\right)$ is the (next-)nearest neighbor coupling. Experimental realizations of such spin chain models are possible and using various analytical and numerical techniques, we present a detailed and quantitative comparison between the usual free electron Kondo model and such spin chain versions of the Kondo problem. For the gap-less $J_{1}-J_{2}$ spin chain two cases are studied, with zero next nearest neighbor coupling, $J_{2}=0$, and with a critical second neighbor coupling, $J_{2}=J_{2}^{c}$. We first focus on the spin chain impurity model with a critical second neighbor antiferromagnetic exchange $J_{2}^{c} \simeq 0.2412$ where a bulk marginal coupling present in the spin chain model for $J_{2}<J_{2}^{c}$ vanishes. There, the usual Kondo physics is recovered in the spin chain model in the low energy regime (up to negligible corrections, dropping as powers of inverse length or energy). At $J_{2}^{c}$ the spin chain model is not exactly solvable and we demonstrate the equivalence to the Kondo problem by comparing Density Matrix Renormalization Group calculations on the frustrated spin chain model with exact Bethe Ansatz calculations of the electronic Kondo problem. We then analyze the nearest-neighbor model $\left(J_{2}=0\right)$ where a new kind of Kondo effect occurs due to the presence of the bulk marginal coupling. This marginal coupling alters slightly the $\beta$-function for the Kondo coupling leading to a slower variation of the Kondo temperature $T_{K}$ with the bare Kondo coupling. In the exact Bethe ansatz solution
\end{abstract}

$\ddagger$ Permanent address: Department of Physics and Astronomy, McMaster University, Hamilton, ON, L8S 4M1, Canada 
of this spin chain impurity model $\left(J_{2}=0\right)$ Frahm and Zvyagin noted this relation as well as the connection to the Kondo problem. Here, by numerically solving the Bethe ansatz equations we provide further evidence for the connection to Kondo physics and in addition we present low temperature Quantum Monte Carlo results for the impurity susceptibility that further support this connection.

PACS numbers: 75.10.Pq,75.20.Hr,75.40.Mg,03.70.+k,04.20.Jb 


\section{Introduction}

The usual Kondo Hamiltonian [1, 2] contains a Heisenberg interaction between an impurity spin and otherwise non-interacting electrons. A simple model takes a free electron dispersion relation and a $\delta$-function Kondo interaction:

$$
H=\int d^{3} r\left[\psi^{\dagger}\left(-\nabla^{2} / 2 m\right) \psi+J_{K} \delta^{3}(\vec{r}) \psi^{\dagger}(\vec{\sigma} / 2) \psi \cdot \vec{S}_{i m p}\right] .
$$

Upon expanding in harmonics, this can generally be reduced to a one-dimensional model of electrons on a semi-infinite line interacting with the impurity spin at the origin [3]. We shall refer to this model as the standard free electron Kondo model (FEKM). A closely related model consists of an open gap-less spin- $\frac{1}{2}$ Heisenberg chain, defined on a semi-infinite line, with one coupling at the end of the chain weaker than the others, described by the following Hamiltonian (see Fig. 1):

$$
\mathcal{H}=J_{1} \sum_{i=1}^{L-1} \vec{S}_{i} \cdot \vec{S}_{i+1}+\mathcal{H}_{i m p}, \quad \mathcal{H}_{i m p}=J_{K}^{\prime} \vec{S}_{i m p} \cdot \vec{S}_{1}
$$

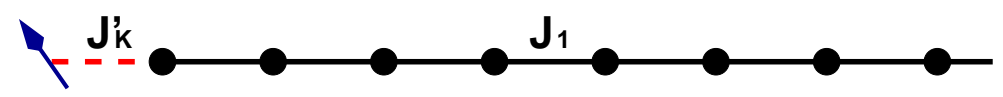

Figure 1. Schematic picture for an open Heisenberg chain coupled to a spin impurity (arrow) at the left boundary via a weak antiferromagnetic exchange $J_{K}^{\prime}$ (dashed bond).

More generally, it is of interest to add a frustrating second neighbor interaction, $J_{2}$, to this gap-less spin Hamiltonian while keeping the open boundary conditions. In this case we arrive at a slightly generalized version of the SCKM:

$$
\begin{aligned}
& \mathcal{H}=J_{1} \sum_{i=1}^{L-1} \vec{S}_{1} \cdot \vec{S}_{i+1}+J_{2} \sum_{i=2}^{L-2} \vec{S}_{i} \cdot \vec{S}_{i+2}+\mathcal{H}_{i m p}, \\
& \mathcal{H}_{i m p}=J_{K}^{\prime} \vec{S}_{i m p} \cdot\left(J_{1} \vec{S}_{1}+J_{2} \vec{S}_{2}\right) .
\end{aligned}
$$

In the following we shall always set $J_{1} \equiv 1$. We refer to both spin chain models,

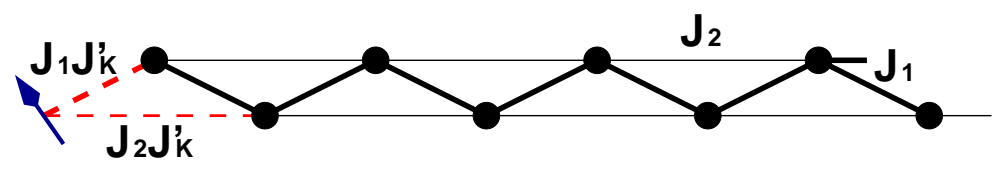

Figure 2. Schematic picture for the open frustrated Heisenberg chain coupled to a spin impurity (arrow) [model (1.3)]. Thin lines show the frustrating second neighbor couplings $J_{2}$.

Eqs. (1.2) and (1.3), as the Spin Chain Kondo Model (SCKM). As $J_{2}$ is increased a critical point $J_{2}^{c} \approx 0.2412$ [4] is reached beyond which the spin chain enters a dimerized phase [5] with a gap and the relation between Eq. (1.3) and Kondo physics no longer holds. 
The purpose of this paper is to explore in some detail the relationship between the FEKM and the SCKM. Such a relationship was pointed out in $[6,7]$ using field theoretical techniques and numerical solutions of finite chains. In [8], hereafter referred to as FZ, it was pointed out that the spin Hamiltonian of Eq. (1.2) (or Eq. (1.3) with $\left.J_{2}=0\right)$ is Bethe ansatz integrable and a number of its properties were determined exactly, establishing some connection with the usual (free electron) Kondo model. However, certain clear differences between the two models are evident from the exact solution. In particular, the characteristic energy scale, the Kondo temperature, behaves exponentially at small Kondo coupling, $T_{K} \propto e^{-c / J_{K}}$ (where $c$ is a constant) in the FEKM but has an unusual exponential square root behavior [8] in the SCKM at $J_{2}=0$, $T_{K} \propto e^{-c / \sqrt{J_{K}^{\prime}}}$. This behavior is likely generic for all $J_{2}<J_{2}^{c}$. However, as we shall see, once we tune $J_{2}$ to the special critical point $J_{2}^{c} \approx 0.2412$ in the SCKM the usual exponential behavior $T_{K} \propto e^{-c / J_{K}^{\prime}}$ is recovered.

Here we will explain the subtle differences in the low energy properties of the FEKM and SCKM using a renormalization group/field theory approach. The differences arise from a marginally irrelevant interaction in the spin chain model, with marginal coupling constant $g$. This is a bulk interaction, which does not occur in the FEKM and is unrelated to the Kondo interaction. At $J_{2}^{c}$ the marginal coupling constant vanishes, $g=0$, and the usual Kondo physics is exactly recovered in the SCKM.

In the strong coupling limit of the usual Kondo model, the impurity spin is 'screened' in the sense that it will form a non-magnetic singlet with the rest of the system. The screening is expected to occur on a characteristic length scale, defining the so called screening cloud:

$$
\xi_{K}=v / T_{K} \propto e^{1 / \lambda_{K}} .
$$

Here $v$ is the characteristic velocity of the low energy excitations. Physical quantities with zero anomalous dimension are expected to show scaling with $\xi_{K} / L$. Strong evidence for the occurrence of Kondo physics in the low-energy sector of the SCKM can therefore be established if estimates, not only of $\xi_{K}$ (or equivalently $T_{K}$ ), but of the entire scaling functions coincide in the SCKM and FEKM. We show that this is clearly the case for the SCKM at $J_{2}=J_{2}^{c}$ while for the SCKM at $J_{2}=0$ the logarithmic corrections arising from the marginal coupling constant $g$ leads to scaling violations and as mentioned above, the divergence of $T_{K}$ and therefore $\xi_{K}$ acquires an unusual exponential square root behavior. Still, the strong coupling limit remains that of a fully screened impurity for $J_{2}=0(g>0)$.

Our conclusions will be confirmed in detail through large-scale numerical calculations. To this end, we have made extensive use of the two known exact Bethe ansatz solutions, for the FEKM $[9,10]$ and for the SCKM at $J_{2}=0[8]$. Numerical results are then obtained by solving the two sets of Bethe ansatz equations (BAE) for finite systems of size $L$ at $T=0$. At finite $T$, we also obtain quantum Monte Carlo (QMC) results for the SCKM at $J_{2}=0$. For general $J_{2}$, and in particular at $J_{2}^{c}$ where the relation between the SCKM and FEKM is most direct, the SCKM is not exactly 
solvable and a sign problem appears in QMC simulations due to the frustrating term $J_{2}$. Fortunately, high precision results for the SCKM at $T=0$ in this regime $\left(J_{2} \neq 0\right)$ can be obtained using Density Matrix Renormalization Group (DMRG) methods.

These results are useful in two ways. First of all, the SCKM has distinct advantages over the free electron version for numerical simulation since the Hilbert space grows much more slowly with system size, $L$, as $2^{L}$ rather than the $4^{L}$ behavior of the free electron model. (On the other hand, the Numerical Renormalization Group technique provides a powerful alternative numerical approach for the free electron model.) In particular, we presented extensive DMRG results on entanglement entropy for the Kondo model using the spin chain version in $[11,12,3]$. In addition, experimental realizations of the SCKM are possible, in particular in muon spin resonance experiments [13]. In experiments on spin chains the coupling $J_{2}$ usually cannot be tuned and a detailed understanding of the relation to the FEKM as $J_{2}$ is varied over experimentally realistic values is therefore of considerable interest.

This paper is organized as follows. In section 2 we briefly review the field theory and renormalization group $(\mathrm{RG})$ treatment of both free electron and spin chain Kondo models. We then study the interplay of the marginal bulk interaction and Kondo interaction in the SCKM, explaining the origin of the exponential square root form of the Kondo temperature using a renormalization group approach. In section 3 we present results on the SCKM with a (bulk) next nearest neighbor interaction, Eq. (1.3), tuned to the critical point, $J_{2}^{c} \approx 0.2412$, separating gap-less and dimerized phases. The presence of the frustrating coupling, $J_{2}$, allows the bulk marginal coupling, $g$ to be tuned and at the critical point, $J_{2}^{c}$, it vanishes, $g=0$, where excellent agreement is obtained with the FEKM. A precise relationship between $J_{K}^{\prime}$ in the SCKM and the Kondo coupling, $\lambda_{K}$, in the FEKM is obtained in this case which is needed for detailed comparisons $[3,12]$. This relation is established in sub-section 3.1. In sub-section 3.2 we then turn to a discussion of the finite-size corrections to the triplet-singlet gap in the FEKM and SCKM (at $J_{2}^{c}$ ) and the resulting scaling with $\xi_{K} / L$. This allows for a convenient way of extracting $\xi_{K}$ and we show that estimates of $\xi_{K}$ and the entire scaling function coincide in the FEKM and SCKM at $J_{2}=J_{2}^{c}$. We end section 3 by deriving the finite-size corrections to the ground-state energy in sub-section 3.3. As previously shown $[3,12]$, a universal term proportional to the Kondo length scale can be identified in the ground-state energy at intermediate values of the Kondo coupling. This allows for an independent and absolute estimate of $\xi_{K}$ in the SCKM in excellent agreement with those obtained from the scaling of the singlet-triplet gap. In section 4 we turn to a discussion of our results for the nearest neighbor SCKM, Eq. (1.2), with $J_{2}=0$ where $g>0$ and logarithmic corrections arising from $g$ are present. In subsection 4.1 we first calculate the impurity susceptibility of the $\operatorname{SCKM}\left(J_{2}=0\right)$ at finite temperature using QMC and compare it to the known result for the FEKM obtained from the BAE. In sub-section 4.2 we discuss the singlet-triplet gap for the finite size system. The result is compared with weak and strong Kondo coupling perturbative results, supplemented by weak coupling perturbation theory in $g$ for the SCKM. This 
is shown to be a convenient quantity for extracting the Kondo screening cloud size, $\xi_{K}$. It compares well to the expected $v_{s} / T_{K}$ where $v_{s}$ is the characteristic velocity. We then discuss the finite size corrections to the ground-state energy for the SCKM with finite $\xi_{K}$ and $g>0$ in sub-section 4.4. We close section 4 by discussing Bethe ansatz results of the $T=0$ magnetization [8], $M(H)$, in sub-section 4.5. We observe that, in addition to the standard scaling function of $H / T_{K}$ which occurs in the FEKM, additional scaling violating terms appear which can be expanded in $g(H)$. Finally, in section 5 we summarize our results by showing $T_{K}\left(J_{K}^{\prime}\right)$ as obtained for the SCKM both at $J_{2}=0$ and $J_{2}^{c}$, and compare to the formula obtained by $\mathrm{FZ}$ and to that obtained from RG calculations in section 2.

\section{The Kondo effect: A one dimensional problem}

\subsection{Low energy theory}

Due to the spherically symmetric free particle dispersion relation of the $3 \mathrm{D}$ model in Eq. (1.1), we may expand the electron annihilation operators in spherical harmonics. Due to the $\delta$-function form of the Kondo interaction, only the $s$-wave harmonic interacts with the impurity. (See, for example, [14] for more details.) Assuming a weak Kondo coupling, we may linearize the dispersion relation near the Fermi momentum, $k_{F}$, yielding the 1D low energy effective Hamiltonian:

$$
H=\frac{v_{F}}{2 \pi} \int_{0}^{\infty} d x\left[i \psi_{L}^{\dagger} \frac{d}{d x} \psi_{L}-i \psi_{R}^{\dagger} \frac{d}{d x} \psi_{R}\right]+v_{F} \lambda_{K} \psi_{L}^{\dagger}(0) \frac{\vec{\sigma}}{2} \psi_{L}(0) \cdot \vec{S}
$$

Here the left and right moving Fermi fields are functions of $\left(v_{F} t+x\right)$ and $\left(v_{F} t-x\right)$ respectively and obey the (unconventionally normalized) equal time anti-commutation relations:

$$
\left\{\psi_{L / R}(x), \psi_{L / R}^{\dagger}(y)\right\}=2 \pi \delta(x-y),
$$

and the boundary condition at the origin:

$$
\psi_{L}(x=0)=-\psi_{R}(x=0) .
$$

[We have adopted the normalization conventions for the fermion fields and the definition of $\lambda_{K}$ used in [14]. $\lambda_{K}=\nu J_{K}$ where $\nu$ is the density of states (per spin) $\nu=k_{F} m /\left(2 \pi^{2}\right)$.]

A convenient way of analyzing the 1D model is to bosonize, since this separates spin and charge degrees of freedom. First we take advantage of the boundary conditions (2.3) and the fact that $\psi_{L / R}(t, x)=\psi_{L / R}\left(v_{F} t \pm x\right)$, to write the right movers as the analytic continuation of the left movers to the negative axis:

$$
\psi_{L}(-x) \equiv-\psi_{R}(x), \quad(x>0) .
$$

Then the Hamiltonian, written in terms of left-movers only, becomes:

$$
H=\frac{v_{F}}{2 \pi} \int_{-\infty}^{\infty} d x i \psi_{L}^{\dagger} \frac{d}{d x} \psi_{L}+v_{F} \lambda_{K} \psi_{L}^{\dagger}(0) \frac{\vec{\sigma}}{2} \psi_{L}(0) \cdot \vec{S}
$$


It is possible to write the kinetic energy in a form quadratic in charge and spin currents:

$$
J_{L}(x) \equiv \psi_{L}^{\alpha \dagger}(x) \psi_{L \alpha}(x), \quad \vec{J}_{L} \equiv \psi_{L}^{\alpha \dagger} \frac{\vec{\sigma}_{\alpha}^{\beta}}{2} \psi_{L \beta}(x) .
$$

(Here repeated indices are summed.) The Hamiltonian becomes:

$$
H=\frac{v_{F}}{2 \pi} \int_{-\infty}^{\infty} d x\left[\frac{1}{4}: J_{L} J_{L}(x):+\frac{1}{3} \vec{J}_{L} \cdot \vec{J}_{L}\right]+v_{F} \lambda_{K} \vec{J}_{L}(0) \cdot \vec{S} .
$$

The spin and charge currents operators may be expressed in terms of spin and charge boson fields and only the spin bosons couple to the impurity, $\vec{S}$. The kinetic energy terms just correspond to free massless (charge and spin) boson kinetic energies.

\subsection{Renormalization group}

For a weak bare coupling, we can estimate $\xi_{K}$ as the length scale at which the effective coupling becomes $O(1)$. From the weak coupling $\beta$-function, the variation of the effective Kondo coupling as we vary the length scale is:

$$
\frac{d \lambda_{K}}{d \ln L}=\beta\left(\lambda_{K}\right)=\lambda_{K}^{2}-\frac{1}{2} \lambda_{K}^{3}+\ldots
$$

Here $\lambda_{K}(L)$ is the effective coupling at length scale $L$. Alternatively, we may identify $L$ with $v_{F} / E$ where $v_{F}$ is the velocity and $E$ is a characteristic energy scale such as a temperature or magnetic field.

We may integrate this equation from a length scale of the order a lattice constant, $a$, up to $\xi_{K}$ with the effective Kondo coupling varying from its bare value $\lambda_{K}^{0}$ to a value $c$, of $O(1)$. Assuming $\lambda_{K}^{0} \ll 1$, this gives approximately:

$$
\xi_{K} \approx\left[a e^{-1 / c} \sqrt{c}\right] \frac{1}{\sqrt{\lambda_{K}^{0}}} \exp \left[1 / \lambda_{K}^{0}\right]
$$

Note that the first factor, in square brackets is simply a constant. Including higher order terms in the $\beta$-function only leads to relatively small corrections of the form:

$$
\xi_{K}=\frac{\text { constant }}{\sqrt{\lambda_{K}}} \exp \left[1 / \lambda_{K}\right]\left[1+O\left(\lambda_{K}\right)\right] .
$$

(Here we have dropped the subscript 0 from $\lambda_{K}$ which refers to the bare value of the Kondo coupling.) We may solve for the effective coupling at scale $L$ in terms of $\xi_{K}$ at weak coupling (i.e. $L \ll \xi_{K}$ ):

$$
\lambda_{K}(L) \approx \frac{1}{\ln \left(\xi_{K} / L\right)}+\frac{1}{2} \frac{\ln \left[\ln \left(\xi_{K} / L\right)\right]}{\ln ^{2}\left(\xi_{K} / L\right)} .
$$

\subsection{Fermi liquid theory}

At low energies and long length scales, the effective Kondo coupling becomes large, and the effective Hamiltonian flows to the strong coupling fixed point. We may think of this fixed point as one where the impurity spin is "screened", i.e. it forms a singlet with a conduction electron. The remaining electrons behave, at low energies and long length scales, as if they were non-interacting, except that they obey a modified boundary 
condition reflecting the fact that they cannot break up the singlet by occupying the same orbital as the screening electron. This modified boundary condition corresponds to a $\pi / 2$ phase shift. Correspondingly in the spin chain Kondo model, the impurity spin gets "adsorbed into the chain" and no longer behaves like a paramagnetic spin at low energies and long distances. The leading corrections to this low energy long distance picture are described by lowest order perturbation theory in the leading irrelevant operator at the strong coupling fixed point. This is an interaction between the remaining conduction electrons, near the screened impurity. (It doesn't involve the impurity itself since it is screened and doesn't appear in the low energy effective Hamiltonian.)

This leading irrelevant operator is $\vec{J}_{L} \cdot \vec{J}_{L}(0)[15,16]$. From $(2.7)$, we see that this is proportional to the spin part of the free electron energy density, $\mathcal{H}_{s, L}(0)$. The energy density has dimensions of (energy)/(length) so the corresponding coupling constant in the effective Hamiltonian must have dimensions of length. On general scaling grounds we expect it to be proportional to $\xi_{K}$. The precise constant of proportionality simply corresponds to giving a precise definition of what we mean by $\xi_{K}$. We adopt the convention:

$$
H_{\text {int }}=-\left(\pi \xi_{K}\right) \mathcal{H}_{s, L}(0)
$$

Here the subscripts $s$ and $L$ are a reminder that this is the spin only part of the energy density for left movers. Note that if we start with a system of length $L$ imposed OBC (with left and right movers), then we can map into a system of periodic length $2 L$ with left movers only. For the purpose of doing first order perturbation theory in $H_{\text {int }}$ for quantities like the susceptibility, specific heat or ground state energy, which are translationally invariant in $0^{\text {th }}$ order, we may replace [14] $H_{\text {int }}$ by:

$$
H_{\text {int }} \rightarrow-\frac{\pi \xi_{K}}{2 L} \int_{-L}^{L} \mathcal{H}_{s, L}(x) .
$$

This is equivalent to a length dependent reduction of the velocity:

$$
v_{F} \rightarrow v_{F}\left[1-\frac{\pi \xi_{K}}{2 L}\right] .
$$

This then implies that the $T=0$ susceptibility, which is $L /\left(2 \pi v_{F}\right)$ in the absence of the Kondo impurity becomes:

$$
\begin{aligned}
\chi \rightarrow \frac{L}{2 \pi v_{F}\left[1-\pi \xi_{K} /(2 L)\right]} & \approx \frac{L}{2 \pi v_{F}}+\frac{\xi_{K}}{4 v_{F}} \\
& =\frac{L}{2 \pi v}+\frac{1}{4 T_{K}} .
\end{aligned}
$$

Thus the zero temperature impurity susceptibility is $1 /\left(4 T_{K}\right)$. It is this form of the impurity susceptibility, simply related to the high temperature, free spin behavior, $1 /(4 T)$, which motivates the definition of $\xi_{K}$ (and hence $T_{K}=v_{F} / \xi_{K}$ ) implied by (2.12). We note that this interaction $H_{i n t}$ is present even in the absence of an impurity, for free fermions but then the coupling constant is of order a lattice constant. Similarly, it is also present for the spin chain with no impurity (i.e. $J_{K}^{\prime}=1$ ) with a coupling 
constant of order a lattice constant. The effect of a weak Kondo coupling is to make this coupling constant large. We emphasize that this precise choice of definition of $T_{K}$ has no physical consequences. The power of Fermi liquid theory is to predict not only the form of low energy quantities but also ratios of various low energy quantities such as impurity susceptibility, impurity specific heat, resistivity, etc., corresponding to various generalized Wilson ratios.

\subsection{Field theory approach to the Spin Chain Kondo Model}

A field theory approach to the spin chain Hamiltonian of Eq. (1.2) is obtained by bosonization. The spin operators have uniform and staggered parts:

$$
\vec{S}_{j} \rightarrow\left[\vec{J}_{L}(a j)+\vec{J}_{R}(a j)\right] /(2 \pi)+(-1)^{j} \text { constant } \cdot \vec{n}(a j),
$$

where $a$ is the lattice constant and $\vec{J}_{L / R}, n$ vary slowly on that scale. The left and right moving current operators are equivalent to the corresponding operators in the free fermion model. On the other hand, the staggered spin density, $\vec{n}$, with a scaling dimension of $1 / 2$, has no counterpart in that model. The low energy effective Hamiltonian is simply that of the spin part of the free electron Hamiltonian,

$$
H_{0}=\frac{v_{s}}{6 \pi} \int_{0}^{L}\left[\vec{J}_{L}^{2}+\vec{J}_{R}^{2}\right]
$$

Here $v_{s}$ is the spin velocity. In addition, there are various irrelevant operators appearing in the effective Hamiltonian. The only one which is important at long distances and low energies is a marginally irrelevant interaction,

$$
H_{\text {int }}=-\frac{g v_{s}}{2 \pi} \int_{0}^{L} \vec{J}_{L} \cdot \vec{J}_{R} .
$$

The bare coupling constant, $g_{0}$, has a positive value of $\mathrm{O}(1)$ but renormalizes to zero at low energies and long lengths scales. As a frustrating next neighbor interaction, $J_{2}$ is turned on $g_{0}$ decreases, passing through zero at a critical coupling, $J_{2} \approx 0.2412$. For still larger $J_{2}, g_{0}$ becomes negative, and hence marginally relevant.

The RG equation for the bulk marginal coupling constant, $g$, is:

$$
\frac{d g}{d \ln L}=\beta(g)=-g^{2}-\frac{1}{2} g^{3}+\ldots
$$

Integrating from a scale, $L_{0}$ of order a lattice constant where the bare coupling has a value $g_{0}>0$ to an arbitrary scale $L$ gives:

$$
\int_{g_{0}}^{g(L)} \frac{d g}{\beta(g)}=\ln \left(L / L_{0}\right) .
$$

It is known that $g(L)$ flows, at large length scales, to the weak coupling region where the expansion of Eq. (2.19) becomes valid. For large L, the integral in Eq. (2.20) is dominated by the small $g$ region giving:

$$
\frac{1}{g(L)}+\frac{1}{2} \ln g(L)=\ln \left(L / L_{0}\right)+\text { constant } \equiv \ln \left(L / L_{1}\right) .
$$


The constant $L_{1}$ cannot, in general, be determined by elementary means. However, if the bare coupling, $g_{0}$ is weak then:

$$
\ln \left(L / L_{1}\right) \approx \ln \left(L / L_{0}\right)+\frac{1}{g_{0}}
$$

so $L_{1} \ll L_{0}$. For the nearest neighbor Heisenberg model, $L_{1}$ is $\mathrm{O}(1)$ and can be determined by comparing to the Bethe ansatz solution (for $J_{K}^{\prime}=1$ ) [17]. In this case, the best choice of $L_{1}$ depends on precisely what quantity is being calculated. In any event, at sufficiently large $L$, where $L \gg L_{1}$, we may approximately solve Eq. (2.21) to obtain:

$$
g(L) \approx \frac{1}{\ln \left(L / L_{1}\right)}-\frac{1}{2} \frac{\ln \left[\ln \left(L / L_{1}\right)\right]}{\ln ^{2}\left(L / L_{1}\right)} .
$$

Note that the $\beta$ functions for the Kondo coupling, $\lambda_{K}(L)$ and bulk marginal coupling, $g(L)$ are the same, to cubic order except for the sign of the quadratic term. Hence the expressions, Eq. (2.11) and (2.23) look similar. There are crucial differences however. Eq. (2.11) is only valid at relatively short distances (high energies) where $L \ll \xi_{K}$; $\lambda_{K}(L)$ grows with increasing $L$. Eq. (2.23) is only valid at long distances (low energies), $L \gg L_{0}$ for the nearest neighbor Heisenberg model; $g(L)$ decreases with increasing $L$.

For $g_{0}<0$, there is a gap of order $\sqrt{\left|g_{0}\right|} e^{-1 /\left|g_{0}\right|}$, corresponding to the spontaneously dimerized phase. The critical point, where $g_{0}=0$ is thus an especially good point for numerical studies since, for $g_{0}>0$ the slowly decreasing marginal coupling constant complicates finite size scaling.

Weakly coupling an impurity spin to a single spin at a generic point in a spin chain yields a model $[6,7]$ with essentially no relation to the standard Kondo model. On the other hand, the model of Eq. (1.2) with the impurity spin coupled at the end of the chain, is quite different. To see this, first consider the limit $J_{K}^{\prime}=g=0$. The open boundary condition leads to:

$$
\vec{J}_{R}(0)=\vec{J}_{L}(0) \propto \vec{n}(0) .
$$

Thus the SCKM is equivalent to the FEKM for some non-trivial constant of proportionality between Kondo couplings, and with the Fermi velocity replaced by the spin velocity, ignoring the irrelevant operator.

The low energy effective Hamitonian of the SCKM Eq. (1.2) has three main terms:

$$
H=\frac{v_{s}}{2 \pi} \int_{-L}^{L} d x \frac{1}{3}\left[\vec{J}_{L}(x)\right]^{2}-\frac{g v_{s}}{2 \pi} \int_{0}^{L} d x \vec{J}_{L}(x) \cdot \vec{J}_{R}(x)+\lambda_{K} v_{s} \vec{J}_{L}(0) \cdot \vec{S} .
$$

The first term, which we denote $H_{0}$, corresponds to a free boson Hamiltonian, the second term is the marginal bulk interaction, and the third term stands for the Kondo like interaction at the boundary with $\lambda_{K} \propto J_{K}^{\prime}$. Using the boundary condition, $\vec{J}_{L}(t, x=0)=\vec{J}_{R}(t, x=0)$ we conclude that we can regard $\vec{J}_{R}(x)$ as the analytic continuation of $\vec{J}_{L}(x)$ to the negative $x$-axis:

$$
\vec{J}_{R}(x)=\vec{J}_{L}(-x) \text {. }
$$


Hence we may write the Hamiltonian as:

$$
H=H_{0}-\frac{g v_{s}}{2 \pi} \int_{0}^{L} d x \vec{J}_{L}(x) \cdot \vec{J}_{L}(-x)+\lambda_{K} v_{s} \vec{J}_{L}(0) \cdot \vec{S} .
$$

When $g=0$ this is the same field theory that describes the FEKM, Eq. (2.7), with $v_{F}$ replaced by $v_{s}$, the spin velocity. A non-zero $g$ leads to some differences, but since $g$ is marginally irrelevant, its effects becomes progressively less important at long distances and low energies.

We now consider the renormalization group equations for the model with both $g$ and $\lambda_{K} \neq 0$. The presence of the Kondo interaction, which is only at the boundary, cannot change the renormalization of the bulk interaction, $g$. On the other hand, the reverse is not true; the bulk marginal interaction has an important effect on the renormalization of the Kondo coupling constant. This can be viewed as resulting from the fact that the boundary operator $\vec{J}_{L}(0)$, appearing in the Kondo interaction, which has a scaling dimension of 1 when $g=0$ picks up an anomalous dimension of first order in $g$. This anomalous dimension was calculated in [18]:

$$
x=1-g+\ldots
$$

[Note that, in [18], $g$ was normalized differently, so that $g_{A Q}=(\sqrt{3} / 4 \pi) g$.] This observation determines the RG equations:

$$
\frac{d \lambda_{K}}{d \ln L}=g \lambda_{K}+\lambda_{K}^{2}+\ldots
$$

To solve (2.29) we may substitute $g(L)$ from (2.23), yielding:

$$
\frac{d \lambda_{K}}{d \ln L}=\frac{\lambda_{K}}{\ln \left(L / L_{1}\right)}+\lambda_{K}^{2}
$$

This differential equation can be solved exactly by defining a new effective coupling constant:

$$
\tilde{\lambda}(L) \equiv \frac{\lambda_{K}(L)}{\ln \left(L / L_{1}\right)}
$$

which obeys the RG equation:

$$
\frac{d \tilde{\lambda}}{d \ln L}=\tilde{\lambda}^{2} \ln \left(L / L_{1}\right)
$$

Setting $\lambda_{K}\left(\xi_{K}\right)=1$, and using Eq. (2.23), one can obtain the renormalized expression for the effective Kondo coupling

$$
\lambda_{K}(L) \simeq \frac{\ln \left(L / L_{1}\right)}{(1 / 2) \ln ^{2}\left(\xi_{K} / L_{1}\right)-(1 / 2) \ln ^{2}\left(L / L_{1}\right)+\ln \left(\xi_{K} / L_{1}\right)} .
$$

This gives, for the Kondo length scale $\xi_{K}$, the unusual dependence on the bare Kondo coupling $\lambda_{K}^{0}$ :

$$
\xi_{K}=L_{1} \exp \left[-c+\sqrt{2 \ln \left(L_{0} / L_{1}\right) / \lambda_{K}^{0}+\ln ^{2}\left(L_{0} / L_{1}\right)+c^{2}}\right],
$$

where $c$ is a positive constant of $\mathrm{O}(1)$. 
Clearly, $\lambda_{K}(L)$ is not a scaling function of $\xi_{K} / L$. Nevertheless, when the bare marginal coupling $g_{0}$ is very small, which is true when $J_{2}$ is tuned close to the critical point, the length scale

$$
L_{1} \sim \exp \left(-1 / g_{0}\right) \ll L_{0}
$$

and then

$$
\lambda_{K} \rightarrow \frac{1}{\ln \left(\xi_{K} / L\right)}
$$

Assuming $\lambda_{K}^{0} \ll 1 / \ln \left(L_{0} / L_{1}\right)$, with a fixed bare marginal coupling $g_{0}$ Eq. (2.34) may be approximated:

$$
\xi_{K} \approx L_{1} \exp \sqrt{2 \ln \left(L_{0} / L_{1}\right) / \lambda_{K}^{0}}
$$

Note that this dependence of $\xi_{K}$ on the Kondo coupling, $\xi_{K} \propto \exp \left[b / \sqrt{\lambda_{K}^{0}}\right]$ (where $b$ is a constant), gives a much shorter Kondo length scale than in the usual Kondo effect which occurs when $g_{0}=0: \xi_{K} \propto \exp \left[1 / \lambda_{K}^{0}\right]$. Also note, from Eq. (2.35) that the limit of zero marginal coupling corresponds to $L_{1} \rightarrow 0$. In this limit, $\ln \left(a / L_{1}\right) \gg 1 / \lambda_{K}^{0}$ so we may Taylor expand the square root in (2.34) giving $\xi_{K} \sim \exp \left[1 / \lambda_{K}^{0}\right]$. However, (2.34) and (2.37) only hold when $\xi_{K} \gg L_{1}$ where $L_{1}$ is roughly the scale at which the effective marginal bulk coupling, $g(L)$ starts to become small.

We expect physical quantities with zero anomalous dimension to be generalized

scaling functions. This means that they can be written as functions of $\xi_{K} / L$ and $g(L)$ only. In general, they should have a Taylor expansion in the renormalized coupling $g(L)$ :

$$
f\left(\lambda_{K}, g, L\right)=\sum_{n=0}^{\infty} g^{n}(L) f_{n}\left(\xi_{K} / L\right) .
$$

The leading term, $f_{0}\left(\xi_{K} / L\right)$ should be the same as in the model with $g=0$, the FEKM. Note that, due to the presence of the $g^{n}(L) \sim 1 / \ln ^{n}(L), f$ is not a pure scaling function in the sense that it does not depend only on $\xi_{K} / L$, but since $g(L) \rightarrow 0$ as $L \rightarrow \infty$, $\xi_{K} / L$ scaling becomes a better and better approximation at large $L$. More generally, the inverse length $1 / L$ in Eq. (2.38) may be replaced by an energy scale such as a magnetic field.

The modified (exponential square root) expression for the dependence of the Kondo temperature on Kondo coupling and the slowly decreasing correction, $g f_{1}, g^{2} f_{2}, \ldots$ are the two main differences between the FEKM and the SCKM. In the following sections we demonstrate the correctness of this assertion by considering various physical quantities.

\section{Kondo effect in the SCKM at $J_{2}^{c}$}

We first discuss the frustrated spin chain model (depicted in Fig. 2) with the Hamiltonian $\mathrm{Eq}$ (1.3). As we have already stressed, in this case the marginal coupling is exactly zero, $g=0$, and the mapping to the FEKM becomes exact, up to strictly irrelevant interactions. Hence, in this case, by determining various constants numerically, it is possible to establish a precise mapping between the low-energy, long-distance sectors of 
the FEKM and SCKM. In sub-section 3.1 we numerically determine $v_{s}$ in the SCKM as well as the constant of proportionality between $J_{K}^{\prime}$ in the SCKM and $\lambda_{K}=\nu J_{K}$ in the FEKM. Then, in sub-section 3.2, we directly compare Bethe ansatz results for the FEKM with DMRG results for the SCKM at $J_{2}^{c}$. Finally, in sub-section 3.3 we show how $\xi_{K}$ at intermediate couplings, $J_{K}^{\prime}$, for the SCKM can be determined from the finite-size scaling of the ground-state energy in the SCKM.

First consider the model with $J_{K}^{\prime}=0$. At a general site, $j$, the low energy degrees of freedom of the spin operators are represented as:

$$
\vec{S}_{j} \approx \frac{1}{2 \pi}\left[\vec{J}_{L}(a j)+\vec{J}_{R}(a j)\right]+(-1)^{j} \text { constant } \times \vec{n}(a j) .
$$

Here $a$ is the lattice spacing and $\vec{n}(a j)$, the alternating part of the spin operators can be written in terms of the spin boson field in a non-linear way. At the end of the chain, due to the open boundary condition, we find that $\vec{n}(x) \rightarrow$ constant $\times \vec{J}_{L}(0)$ and therefore:

$$
\vec{S}_{2}+J_{2} \vec{S}_{3} \approx C \vec{J}_{L}(0)
$$

where $C$ is a non-universal constant, depending on the second neighbor coupling, $J_{2}$ in the Hamiltonian. ( $C$ has dimensions of inverse length, and is proportional to the inverse lattice spacing.) Now including a weak coupling, $J_{K}^{\prime}$ to the first spin, the low energy effective Hamiltonian becomes the usual FEKM with the replacements:

$$
v_{F} \rightarrow v_{s}, \quad v_{F} \lambda_{K} \rightarrow C J_{K}^{\prime} .
$$

\subsection{Numerical parameters for the spin chain problem}

Velocity of excitations: In order to make the above correspondence quantitative, one needs to determine exactly the spin velocity $v_{s}$ at the critical point $J_{2}=J_{2}^{c}$. We can use the fact that the finite size scaling of the ground state energy for the uniform SCKM with $J_{K}^{\prime}=1$ is simply given by [19]

$$
E_{0}(L)=\epsilon_{0} L+\epsilon_{1}-\frac{\pi c v_{s}}{24 L}+\frac{a_{2}}{L^{2}}+\frac{a_{3}}{L^{3}}+O\left(1 / L^{4}\right) .
$$

$\epsilon_{0}, \epsilon_{1}$ and $a_{2}, a_{3}$ are non-universal numbers, whereas the $1 / L$ term is universal, proportional to the central charge $\left(c=1\right.$ here) and the spin velocity $v_{s}$. Note that, $v_{s}$ will depend on $J_{2}$ and even though it is known analytically from the Bethe ansatz solution available at $J_{2}=0$ we need to determine it at $J_{2}^{c}$ where no exact results are available. DMRG results for $E_{0}(L)$, computed on chains up to $L=1000$ sites, are shown in Fig. 3 and fitted to Eq. (3.4). We have checked that fitting with a $M^{\text {th }}$-order polynomial form in $1 / L$ does not change the estimate of $v_{s}$ whenever $M \geq 3$. Thus we found for the spin velocity

$$
v_{s}=1.174(1) \text {. }
$$

This is in good agreement with previous estimates [6].

Kondo coupling: Of course, to predict $\xi_{K}$ for the spin chain model we need to exploit Eq. (3.3) to relate $\lambda_{K}$ to $J_{K}^{\prime}$, the weak coupling of the spin at the end of the chain, where the constant $C$ is determined by (3.2). We emphasize that this constant $C$ 


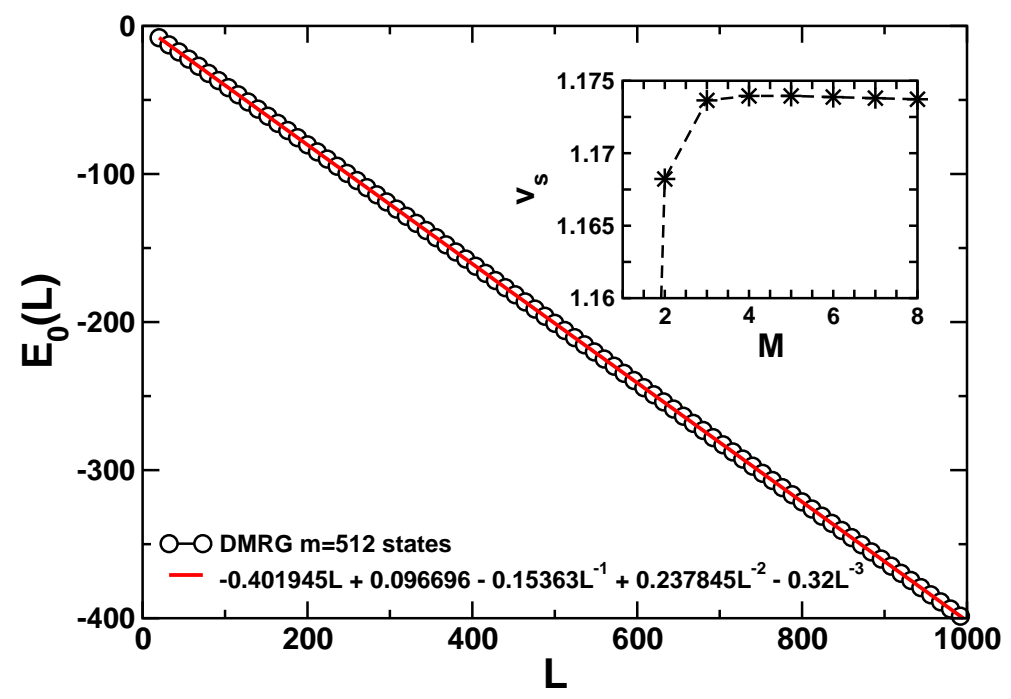

Figure 3. Finite size scaling of the ground state energy $E_{0}(L)$ of the SCKM at the critical second neighbor coupling $J_{2}^{c}=0.2412$ shown for chains up to $L=1000$ sites. DMRG results obtained keeping $m=512$ states (open symbols) are compared to the finite size scaling form (3.4) up to third order (full line). In the inset, $v_{s}$ obtained from polynomial fits of the form $E_{0}(L)=\epsilon_{0} L+\epsilon_{1}-\pi v_{s} /(24 L)+\sum_{i=2}^{M} a_{i} / L^{i}$ is plotted versus $M$.

can be determined in the theory with zero Kondo coupling, $J_{K}^{\prime}=0$ and we can therefore study correlations in chains of even length. A convenient way of determining $C$ is to measure a long-distance correlation function in the spin chain and compare it to the corresponding correlation function in the continuum field theory. The simplest choice appears to be the end-to-end equal time correlation function:

$$
\left\langle\left(\vec{S}_{2}+J_{2} \vec{S}_{3}\right) \cdot\left(\vec{S}_{L}+J_{2} \vec{S}_{L-1}\right)\right\rangle \approx C^{2}\left\langle\vec{J}_{L}(0) \cdot \vec{J}_{L}(L)\right\rangle .
$$

The current correlation function is simply that of the free fermion model with the current defined in Eq. (2.6) and the fermion fields normalized as in Eq. (2.2). For an infinite chain this implies:

$$
\left\langle\vec{J}_{L}(r) \cdot \vec{J}_{L}\left(r^{\prime}\right)\right\rangle=-\frac{3}{2\left(r-r^{\prime}\right)^{2}} .
$$

However, we need the correlation function for a finite strip. Using the fact that the open boundary conditions on a finite strip of length $L$ are equivalent to periodic boundary conditions for left movers only on a circle of circumference $2 L$, we obtain:

$$
\left\langle\vec{J}_{L}(r) \cdot \vec{J}_{L}\left(r^{\prime}\right)\right\rangle=-\frac{3}{\left(8 L^{2} / \pi^{2}\right) \sin ^{2}\left[\pi\left(r-r^{\prime}\right) / 2 L\right]},
$$

giving the end-to-end correlation function:

$$
\left.\left\langle\vec{J}_{L}(0) \cdot \vec{J}_{L} L\right)\right\rangle=-\frac{3 \pi^{2}}{8 L^{2}} .
$$

As shown in Fig. 4 this correlation function can be determined very accurately using DMRG methods, from which we determine:

$$
\left\langle\left(\vec{S}_{2}+J_{2} \vec{S}_{3}\right) \cdot\left(\vec{S}_{L}+J_{2} \vec{S}_{L-1}\right)\right\rangle \rightarrow-\frac{3 \times 0.891462}{L^{2}} .
$$




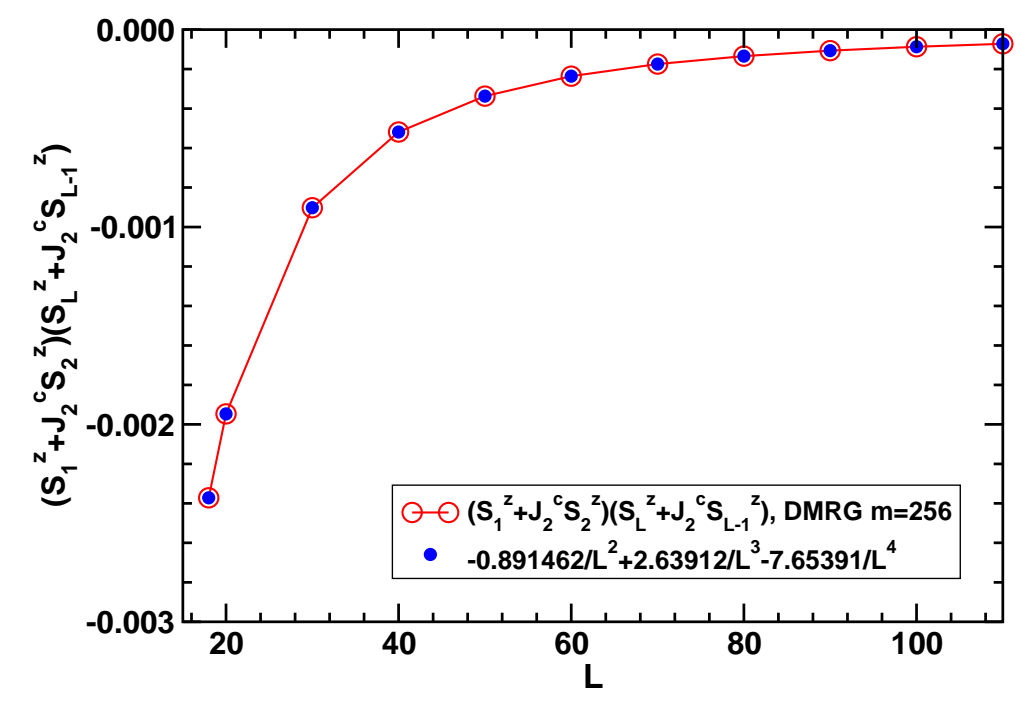

Figure 4. DMRG results keeping $m=256$ states for $\left\langle\left(\vec{S}_{2}^{z}+J_{2} \vec{S}_{3}^{z}\right) \cdot\left(\vec{S}_{L}^{z}+J_{2} \vec{S}_{L-1}^{z}\right)\right\rangle$ for the SCKM at $J_{2}^{c}$ (open circles). Results are shown for $L=18,20,30 \ldots 110$. The solid circles indicates a polynomial fit with the leading term of the form $-0.891462 \mathrm{~L}^{-2}$.

From Eqs. (3.6), (3.9) and (3.10) we obtain:

$$
0.891462=\frac{\pi^{2} C^{2}}{8}, \quad C=0.850054
$$

From (3.3), using $v_{s} \approx 1.174(1)$, we finally obtain the proportionality constant between Kondo couplings in spin chain and fermion models, $J_{K}^{\prime}$ and $\lambda_{K}$ :

$$
J_{K}^{\prime}=\left(v_{s} / C\right) \lambda_{K}=1.38 \lambda_{K} .
$$

\subsection{The triplet-singlet gap and Bethe ansatz results for the FEKM versus DMRG} results on the $S C K M$ at $J_{2}^{c}$

A convenient diagnostic for testing the correspondence between the FEKM and the SCKM at $J_{2}^{c}$ is the finite size behavior of the gap between the singlet ground state and lowest triplet exited state (for even $L$ ). Let us first consider the models with no Kondo coupling. Thus we consider either a free fermion chain or a Heisenberg spin chain with open boundary conditions. Noting that the allowed wave-vectors are $k \approx \pi n / L$, we see that the excitation energy of a triplet particle-hole excitation in the free fermion chain is:

$$
\Delta_{S T}=\frac{\pi v_{s}}{L} .
$$

The same result holds for the spin chain with open boundary conditions [6]. The extension of this result for $\xi_{K} \ll L$, is easily obtained from Fermi liquid theory, by the usual replacement Eq. (2.14):

$$
\Delta_{S T} \rightarrow \frac{\pi v_{s}}{L}\left[1-\frac{\pi \xi_{K}}{2 L}\right]
$$




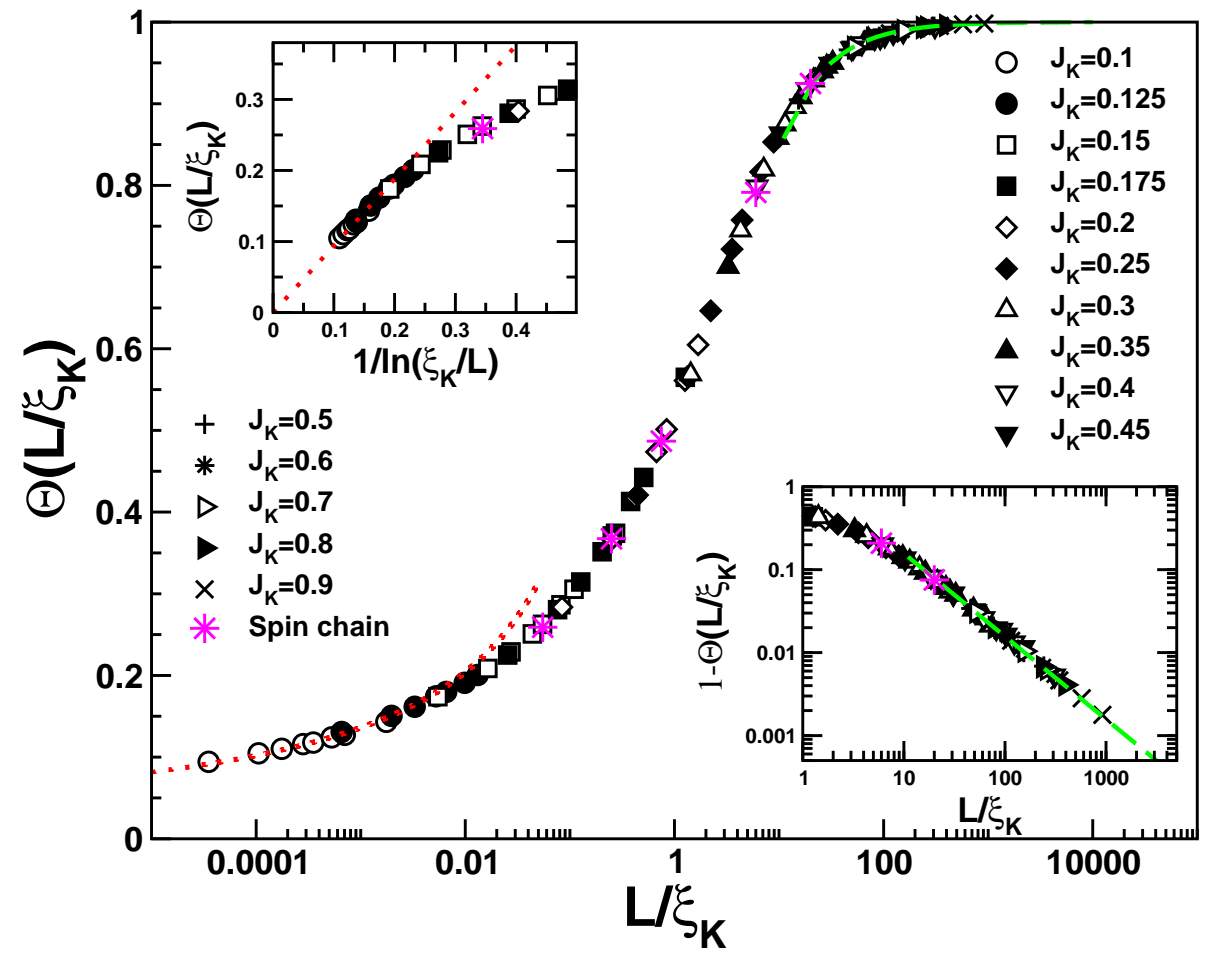

Figure 5. Scaling function $\Theta\left(L / \xi_{K}\right)$ (see Eq. (3.16)) obtained from the Bethe Ansatz solution of the one-channel FEKM Hamiltonian [1] with various values for the Kondo interaction strength $J_{K}$, as indicated on the plot. The scaling plot is constructed using the strong coupling limit curve [long-dashed green line Eq. (3.14)] as a support for the rest of the collapse. The spin chain model (1.3) at $J_{2}^{c}$ results (star) are adjusted to collapse onto the universal curve. Left inset: in the weak coupling regime, the ratio $\Theta \sim 1 / \ln \left(\xi_{K} / L\right)$, as show by the linear fitting dotted line. Right inset: in the strong coupling regime, the FLT result $\Theta=1-\frac{\pi \xi_{K}}{2 L}$ describes perfectly the behavior (long dashed line).

In the weak coupling limit we may calculate $\Delta_{S T}$ in perturbation theory in the Kondo coupling, $\lambda_{K}$. We consider a spin chain with an even number of sites, or equivalently a free fermion chain with an odd number of fermions, coupled to the impurity spin so that the ground state is a spin singlet. In the limit of zero Kondo coupling the ground state of the non-interacting chain is a spin doublet. First order perturbation theory in $\lambda_{K}$ couples this doublet to the impurity spin with a coupling constant $\pi v_{s} / L$, giving a singlet-triplet splitting of $\Delta_{S T} \approx\left(\pi v_{s} / L\right) \lambda_{K}$. We expect that, as usual, higher order corrections will replace $\lambda_{K}$ by its renormalized value at scale $L$, Eq. (2.11), giving:

$$
\Delta_{S T} \rightarrow \frac{\pi v_{s}}{L} \frac{1}{\ln \left(\xi_{K} / L\right)}
$$

Hence, in general we expect the quantity

$$
\Theta \equiv \frac{L}{\pi v_{s}} \Delta_{S T}
$$

to be a universal scaling function of $L / \xi_{K}$. The same function should occur for the FEKM and for the SCKM up to higher order corrections from irrelevant operators. 


\begin{tabular}{|c||ccccc|}
\hline$J_{K}^{\prime}$ & 0.6 & 0.45 & 0.3 & 0.25 & 0.2 \\
\hline$\xi_{K}$ & 5 & 17 & 130 & 400 & 1800 \\
\hline
\end{tabular}

Table 1. Kondo length scale estimates for the SCKM at $J_{2}^{c}$, Eq. (1.3).

Introduced 25 years ago, independently by Andrei [9] and Wiegman [10], the exact diagonalization of the Kondo Hamiltonian (2.5) based on the Bethe Ansatz provides a powerful method to access several physical observables like the uniform susceptibility, the field induced magnetization, the specific heat, the thermodynamic entropy. Similarly to what was previously done using the Bethe Ansatz solution of the unfrustrated chain, one can add an external magnetic field along the $z$-axis $\vec{B}=B \vec{e}_{z}$, which couples to the total spin operator $S_{\text {tot }}^{z}$. Then one can solve numerically for finite length chains the Bethe Ansatz equations $[9,10]$ in any $S_{\text {tot }}^{z}$ sector and compute the singlet triplet excitation gap.

We have calculated $\Theta\left(L / \xi_{K}\right)$ for the FEKM by solving the set of coupled Bethe Ansatz equations for various values of the Kondo coupling $J$, where the convention of Ref. [1] was followed: $J=\frac{\pi}{2} \lambda_{K}$. In Fig. 5 are shown the results for the ratio $\Theta\left(L / \xi_{K}\right)$, successfully compared with the strong and weak coupling predictions Eqs. (3.14) and (3.15).

We have also compared these results to DMRG calculations performed on the Kondo spin chain model with $J_{2}=J_{2}^{c},(1.3)$ for $J_{K}^{\prime}=0.2,0.25,0.3,0.45,0.6$. The ratio $\Theta$ is determined for $L=100$ sites and the DMRG data points (shown as $\star$ ) have been superimposed on the Bethe Ansatz results on Fig. 5, adapting $L / \xi_{K}\left(J_{K}^{\prime}\right)$ in order to get a good collapse. The resulting values for the Kondo length scales are listed in table 1.

The fact that the numerical estimates of not only the length scale $\xi_{K}$, but of the entire scaling function coincide in the FEKM and the SCKM at $J_{2}=J_{2}^{c}$ is very strong independent evidence for the occurrence of Kondo physics in the low-energy sector of the SCKM at $J_{2}=J_{2}^{c}$.

\subsection{Finite size scaling of the ground state energy in the SCKM at $J_{2}^{c}$}

One way of estimating $\xi_{K}$, in the case $\xi_{K} \ll L$, is from a universal correction to the ground state energy. This may be calculated from the Fermi liquid theory Hamiltonian of (2.12). It is well known that the ground state energy of the open spin chain has the form:

$$
E_{G S}=e_{0}+e_{1} L-\frac{\pi v_{s}}{24 L}+\ldots
$$

where $e_{0}$ and $e_{1}$ are non-universal but the third term is universal, depending only on $v_{s}$ and ... indicates terms that drop off faster with $L$. We may include an additional boundary term from first order perturbation in the FLT interaction of (2.12) by replacing $v_{s}$ by its shifted value in (2.14), giving:

$$
\delta E_{G S}=\pi^{2} v_{s} \xi_{K} /\left(48 L^{2}\right) .
$$


We emphasize that such a $1 / L^{2}$ term is always present, even for the uniform chain with open boundary conditions $\left(J_{K}^{\prime}=1\right)$ but that the factor of $\xi_{K}$ is of order the lattice spacing in that case. This term obtains a large coefficient at weak Kondo coupling. Combining these contributions we find for the ground-state energy:

$$
E_{\mathrm{GS}}\left(L, J_{K}^{\prime}\right)=e_{0}\left(J_{K}^{\prime}\right)+e_{1} L-\frac{\pi v_{s}}{24 L}+\left(e_{2}+\frac{\pi^{2} v_{s} \xi_{K}}{48}\right) \frac{1}{L^{2}} .
$$

Please note that, while the constants $e_{1}, e_{2}$ describe bulk behavior and hence are independent of $J_{K}^{\prime}$, the surface term $e_{0}$ does depend on $J_{K}^{\prime}$. Secondly, the difference between $e_{2}$ and $\xi_{K}\left(J_{K}^{\prime}=1\right)$ is largely a matter of convention. Thirdly, this expression for the ground-state energy neglects logarithmic corrections arising from the bulk marginal operator, $g$, assumed to be zero since $J_{2}=J_{2}^{c}$. The more general case with $J_{2}<J_{2}^{c}, g>0$ will be considered in section 4.4 .

Using DMRG results for the ground-state energy we now attempt to determine $\xi_{K}\left(J_{K}^{\prime}\right)$ using Eq. (3.19). As outlined in Appendix B we can in this case eliminate the 3 first terms in Eq. (3.19) by using a form of Richardson extrapolation. We then arrive at:

$$
\begin{aligned}
E^{(3)}\left(L, J_{K}^{\prime}\right) & =\left(e_{2}+\frac{\pi^{2} v_{s} \xi_{K}}{48}\right) c_{2}^{3}(L)+\mathcal{O}\left(L^{-1}\right) \\
& =D c_{2}^{3}(L)+\mathcal{O}\left(L^{-1}\right)
\end{aligned}
$$

where the coefficient $c_{2}^{3}(L)$ is known. Plotting $E^{(3)} / c_{2}^{3}$ should then yield the desired constant, $D$. Plots of $E^{(3)} / c_{2}^{3}(L)$ versus $1 / L$ are shown in Fig. 6. For $J_{K}^{\prime}=0.6,0.8,1.0$

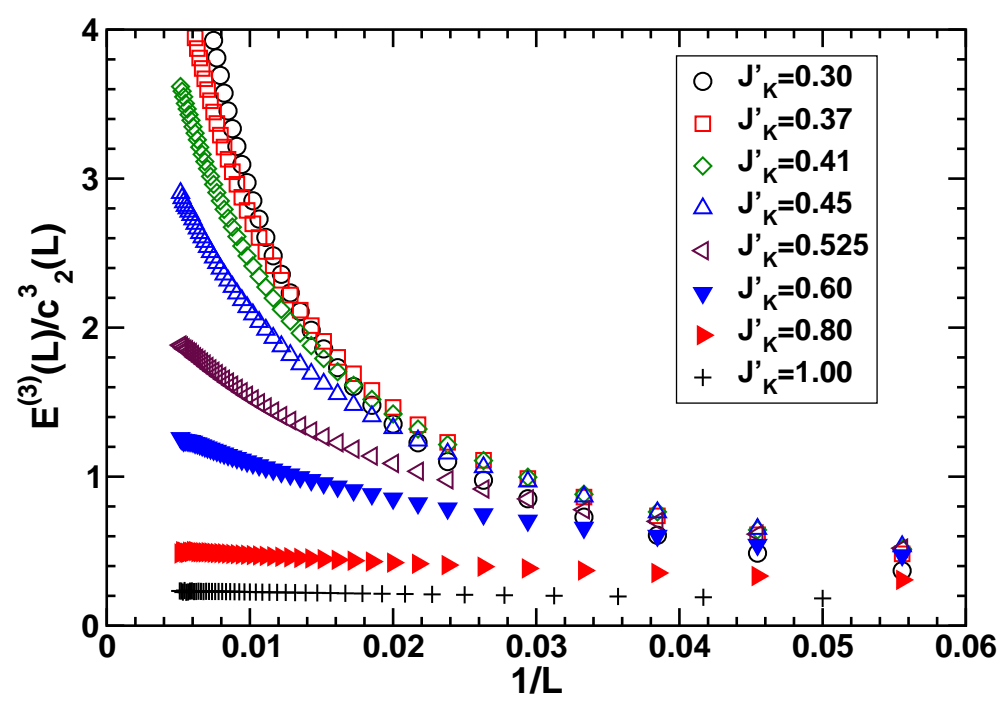

Figure 6. DMRG results keeping $m=256$ states $\left(m=512\right.$ for $\left.J_{K}^{\prime}=1\right)$ for $E^{(3)}\left(L, J_{K}^{\prime}\right) / c_{2}^{3}(L)$ for the SCKM at $J_{2}^{c}$ for a range of $J_{K}^{\prime}$ plotted versus $1 / L$. For the larger $J_{K}^{\prime}$ the results quickly approach a constant as $1 / L \rightarrow 0$. Results are obtained from the ground-state energy for systems with even size.

it is easy to extract the constant $D$ using a simple polynomial fit. However, for smaller 


\begin{tabular}{|c||cccccccc|}
\hline$J_{K}^{\prime}$ & 1.000 & 0.800 & 0.600 & 0.525 & 0.450 & 0.410 & 0.370 & 0.300 \\
\hline$D$ & 0.24093 & 0.54043 & 1.4853 & 2.5426 & 4.76 & 7.14 & 11.5 & $\sim 35$ \\
\hline$\xi_{K}$ & $\equiv 0.65$ & 1.89 & 5.81 & 10.19 & 19.4 & 29.3 & 47 & $\sim 145$ \\
\hline
\end{tabular}

Table 2. The numerically determined values for $D$ for the SCKM at $J_{2}^{c}$ and the resulting $\xi_{K}$.

$J_{K}^{\prime}$ we have found it necessary to fit to a $(2,2)$ rational polynomial to improve the extrapolation. For $J_{K}^{\prime}$ smaller than $J_{K}^{\prime}=0.3$ it is no longer possible to extrapolate using rational polynomials and a reliable numerical determination of $D$ becomes impossible.

As mentioned above, we expect $\xi_{K}$ to be $\mathcal{O}(1)$ for $J_{K}^{\prime}=1$. In previous work $[3,12]$ we used Fermi liquid theory to determine $\xi_{K}\left(J_{K}^{\prime}=1\right)=0.65$ and here we use this result to calibrate our data. In particular, we use this result to determine the coefficient $e_{2}$ using our previous estimate for the velocity, $v_{s}$, finding $e_{2}=0.0841$. The remaining $\xi_{K}$ are then trivial to obtain. In table 2 we list $D\left(J_{K}^{\prime}\right)$ along with the resulting $\xi_{K}$. These independent (and absolute) estimates of $\xi_{K}$ very nicely agree with the ones previously obtained in table 1 clearly establishing that the same length scale $\xi_{K}$ can be extracted from the scaling of the ground-state energy and the singlet-triplet gap as one would expect from Kondo physics.

\section{Kondo effect in the nearest neighbor SCKM, $J_{2}=0$}

We now turn to a discussion of the nearest neighbor SCKM, Eq. (1.2), in the absence of any second neighbor coupling $\left(J_{2}=0\right)$. As outlined, the bulk marginal coupling in the SCKM, $g$, is in this case non-zero, $g>0$. An exact Bethe ansatz solution of this model was developed by FZ [8]. We start by showing QMC results for this non-frustrated model, before presenting BA results for the singlet triplet gap. Then we discuss the connections with the usual Kondo physics.

\subsection{Impurity susceptibility}

We now want to compute the spin susceptibility of the SCKM defined in Eq. (1.2). We take advantage of the non frustrated nature of this antiferromagnetic spin chain model to perform large scale QMC simulations using the Stochastic Series Expansion of the partition function in a loop algorithm framework (see Ref. [20] for details).

4.1.1. Quantum Monte Carlo results While the QMC simulations are done on finite size systems, on can still define the impurity susceptibility by

$$
\chi_{\mathrm{imp}}=\lim _{L \rightarrow \infty}\left[\chi\left(L+1, J_{K}^{\prime}\right)-\chi\left(L, J_{K}^{\prime}=1\right)\right],
$$

where $L+1$ corresponds to a system of $L$ spins in the bulk coupled to 1 boundary impurity with $J_{K}^{\prime}$, and $L$ corresponds to a pure chain of $L$ spins (see Fig. 1). The QMC simulations have been carried out for $L=512$ at low temperatures for various Kondo 
exchange couplings, $J_{K}^{\prime}$. While we are not in the thermodynamic limit $L \rightarrow \infty$, we still expect for such a rather big system size that one can capture all essential features of the Kondo physics present in the spin chain model. Results for $\chi\left(513, J_{K}^{\prime}\right)$ are displayed in Fig. 7 where a clear upturn is visible at low temperature.

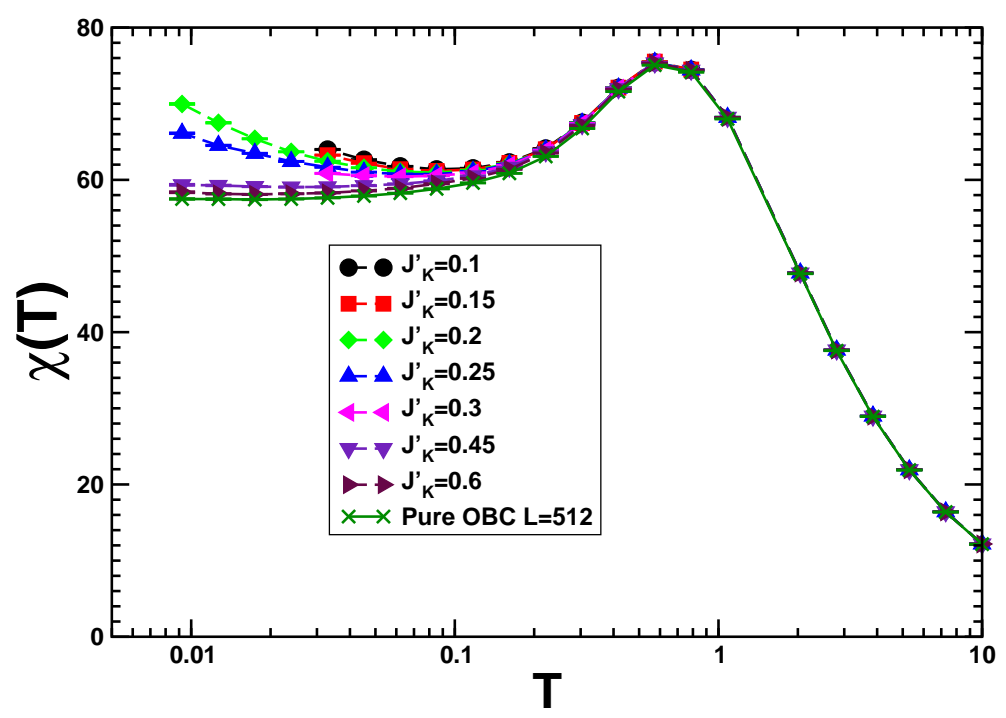

Figure 7. Total susceptibility $\chi(T)$ obtained by Quantum Monte Carlo simulations of the $\operatorname{SCKM}\left(J_{2}=0\right)$ with chains of length $L=512$ coupled to an impurity spin with various Kondo couplings $J_{K}^{\prime}$ indicated as various symbols on the plot. Results for a pure open chain are also shown (solid curve).

As in the usual Kondo problem, one expects the impurity spin to be essentially free at very high temperatures $T \gg T_{K}$, leading to a Curie-like divergence $\sim 1 / T$. On the other hand, when the temperature is decreased, the effective Kondo coupling starts to grow and the impurity eventually becomes screened when $T \ll T_{K}$, forming a strongly entangled spin singlet with the bulk, and thus resulting in an absence of features in the total susceptibility.

4.1.2. Scaling properties and Kondo temperature As in the FEKM, one might expect the impurity susceptibility to be a scaling function of $T / T_{K}$ up to logarithmic corrections coming from the bulk marginal operator $g$. More precisely, weak and strong coupling regimes are characterized by the following behaviors:

$$
4 T_{K} \times \chi_{\mathrm{imp}} \rightarrow\left\{\begin{array}{rl}
T_{K} / T & \text { if } T \gg T_{K} \\
1 & \text { if } T \ll T_{K} .
\end{array},\right.
$$

In the weak coupling regime, the lowest order perturbative expansion

$$
\chi_{\text {imp }}=\frac{1}{4 T}\left(1-1 / \ln \left(T / T_{K}\right)+\ldots\right),
$$

has been used to start building the scaling plot for $T_{K} \times \chi_{\mathrm{imp}}$, as shown in Fig. 8 where the data of Fig. 7 have been used to compute $\chi_{\text {imp. }}$ Step by step, the temperature axis 
has been rescaled with the Kondo temperature $T_{K}$ like $T \rightarrow T / T_{K}$ in order to produce the best collapse of the data onto a curve which reproduces the expected behavior for the usual Kondo problem (see Ref. [2] for instance). Ignoring scaling violations arising from the bulk marginal coupling (which we expect to be small), one expects the scaling plot shown in Fig. 8 to give a rather good estimate of the Kondo temperature $T_{K}$ for the Heisenberg spin chain. The resulting estimates for $T_{K}$ (and thus $\xi_{K}=v_{s} / T_{K}$ with $v_{s}=\pi / 2$ for the Heisenberg chain) are listed in the table 3, and plotted in the inset of Fig. 8. One sees the Kondo energy scale $T_{K}$ going to zero exponentially when $J_{K}^{\prime} \rightarrow 0$. We have successfully compared these estimates to the FZ form [8]

$$
T_{K} \propto \exp \left(-\pi \sqrt{1 / J_{K}^{\prime}-1}\right)
$$

which in the limit of small Kondo coupling $J_{K}^{\prime}$ is equivalent to the exponential squareroot form Eq. (2.37) derived in the previous section from the RG.

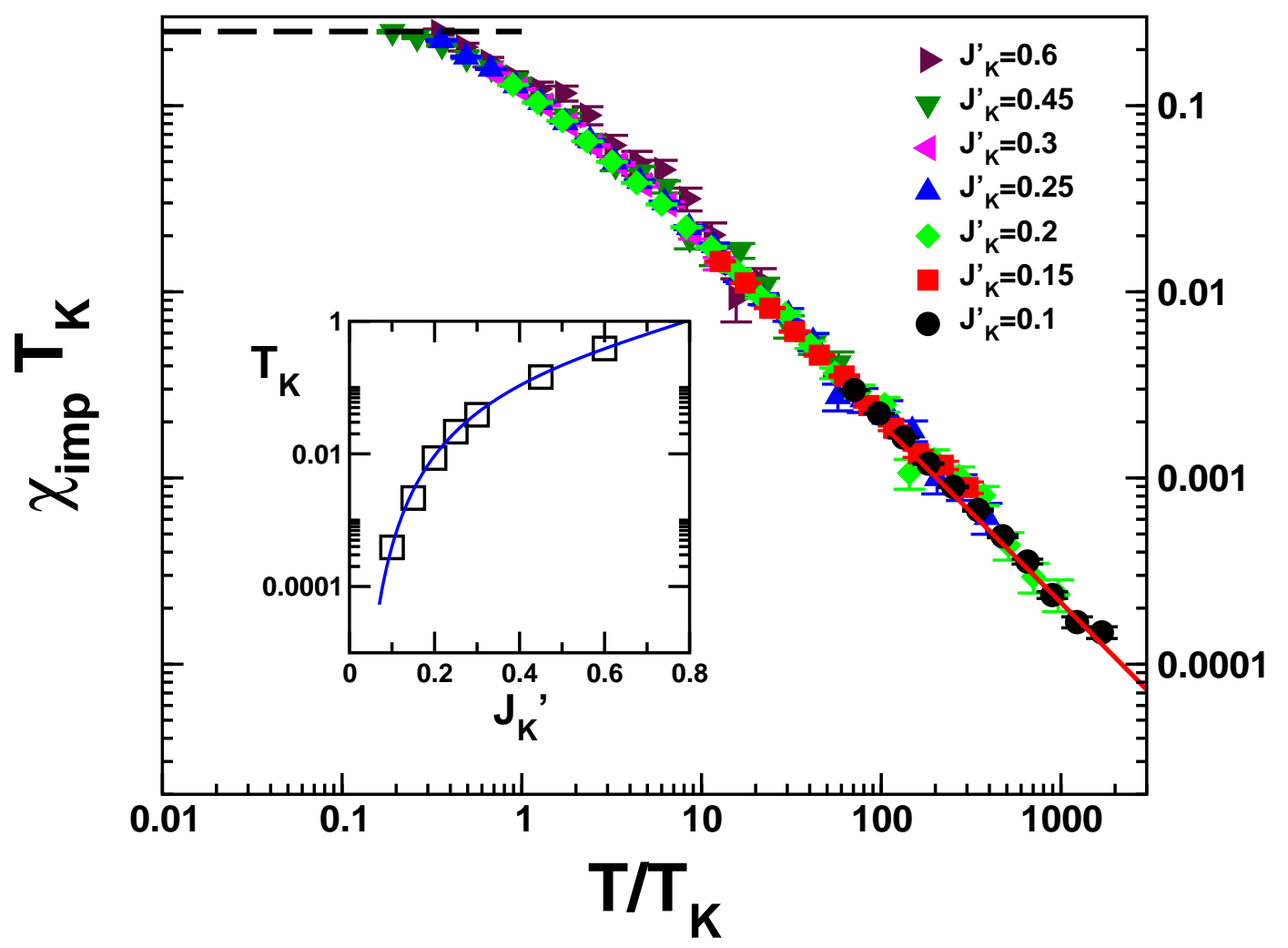

Figure 8. Impurity susceptibility $\chi_{\text {imp }}$ obtained by Quantum Monte Carlo calculations of the SCKM $\left(J_{2}=0\right)$ with chains of length $L=512$ coupled to an impurity spin with various Kondo couplings $J_{K}^{\prime}$ indicated on the plot. The collapse for $\chi_{\mathrm{imp}} \times T_{K}$ was obtained starting from the weak coupling regime with the curve $\chi_{\mathrm{imp}}\left(T \gg T_{K}\right)=0.25 / T\left(1-1 / \ln \left(T / T_{K}\right)\right.$ (red line on the right) serving as a support for the rest of the collapse. The strong coupling limit $\chi_{\text {imp }}\left(T \ll T_{K}\right)=0.25 / T_{K}$ is shown by the black horizontal dashed line on the right. Inset: Kondo temperature estimates obtained from the collapse on the main panel plotted versus the coupling $J_{K}^{\prime}$. The solid curve is the expression Eq. (4.4) with a prefactor $\simeq 5$. 


\begin{tabular}{|r||r|r|r|r|r|r|r|}
\hline$J_{K}^{\prime}$ & 0.1 & 0.15 & 0.2 & 0.25 & 0.3 & 0.45 & 0.6 \\
\hline$T_{K} \sim$ & 0.00039 & 0.0086 & 0.022 & 0.039 & 0.0144 & 0.1 & 0.388 \\
\hline$\xi_{K} \sim$ & 4040 & 727 & 182 & 73 & 40 & 11 & 4 \\
\hline
\end{tabular}

Table 3. Kondo temperature $T_{K}$ and the associated Kondo length scale $\xi_{K}=\pi /\left(2 T_{K}\right)$ for the $\operatorname{SCKM}\left(J_{2}=0\right)$, Eq. (1.2), with a Kondo coupling $J_{K}^{\prime}$. The values are estimated from the impurity susceptibility data (see Fig. 8).

One technical remark has to be mentioned about the QMC data for $\chi_{\text {imp. }}$ At the strong coupling fixed point it is quite difficult to simulate the system at very low temperature $T \ll T_{K}$ and in the scaling limit $T \gg v_{s} / L$ at the same time. Finally, as briefly mentioned above, we expect that the bulk marginal interaction will lead to small corrections to the susceptibility.

\subsection{Bethe ansatz solution}

We start from an open Heisenberg chain with a boundary spin impurity Eqs. (1.2) and an external magnetic field $\vec{B}=B \overrightarrow{e_{z}}$

$$
\mathcal{H}=\sum_{i=1}^{L-1} \vec{S}_{i} \cdot \vec{S}_{i+1}+J_{K}^{\prime} \vec{S}_{i m p} \cdot \vec{S}_{1}-\sum_{i=1}^{L} B S_{i}^{z} .
$$

This model has been shown to be integrable [8] and allows a Bethe Ansatz solution which, in the case where the impurity spin $S_{i m p}=1 / 2$, reads

$$
\left[e_{1}\left(\Lambda_{j}\right)\right]^{2 L} e_{1}\left(\Lambda_{j}+\tilde{\lambda}_{K}\right) e_{1}\left(\Lambda_{j}-\tilde{\lambda}_{K}\right)=\prod_{k=1, k \neq j}^{M} e_{2}\left(\Lambda_{j}-\Lambda_{k}\right) e_{2}\left(\Lambda_{j}+\Lambda_{k}\right) \text {. }
$$

$M$ is the number of down spins, the function $e_{n}(x)=\frac{x+n i / 2}{x-n i / 2}$ and the impurity coupling constant $J_{K}^{\prime}$ is related to $\tilde{\lambda}_{K}$ by

$$
J_{K}^{\prime}=\frac{1}{1+\tilde{\lambda}_{K}^{2}} \text {. }
$$

Note that the $\tilde{\lambda}_{K}$ defined here is not the same as the $\lambda_{K}$ defined in Sec. II. It is more convenient to re-write Eq. (4.6) as

$$
\begin{aligned}
{\left[\frac{\Lambda_{j}+\frac{i}{2}}{\Lambda_{j}-\frac{i}{2}}\right]^{2 L}\left[\frac{\Lambda_{j}+\tilde{\lambda}_{K}+\frac{i}{2}}{\Lambda_{j}+\tilde{\lambda}_{K}-\frac{i}{2}}\right] } & {\left[\frac{\Lambda_{j}-\tilde{\lambda}_{K}+\frac{i}{2}}{\Lambda_{j}-\tilde{\lambda}_{K}-\frac{i}{2}}\right]=} \\
& \prod_{k=1, k \neq j}^{M}\left[\frac{\Lambda_{j}+\Lambda_{k}+i}{\Lambda_{j}+\Lambda_{k}-i}\right]\left[\frac{\Lambda_{j}-\Lambda_{k}+i}{\Lambda_{j}-\Lambda_{k}-i}\right]
\end{aligned}
$$

Then we get

$$
\begin{aligned}
2 L \theta\left(2 \Lambda_{j}\right)+\theta\left(2 \Lambda_{j}+2 \tilde{\lambda}_{K}\right)+ & \theta\left(2 \Lambda_{j}-2 \tilde{\lambda}_{K}\right)= \\
& \sum_{k=1, k \neq j}^{M} \theta\left(\Lambda_{j}+\Lambda_{k}\right)+\theta\left(\Lambda_{j}-\Lambda_{k}\right)-\pi I_{j},
\end{aligned}
$$


where $\theta(x)=\arctan (x)$, the $I_{j}$ are all integers and $j=1, \ldots, M$. The total energy in an external magnetic field $B$ can be expressed [8]

$$
E(M)=\frac{L-1}{4}+\frac{J_{K}^{\prime}}{4}-\frac{1}{2} \sum_{j=1}^{M} \frac{1}{\Lambda_{j}^{2}+1 / 4}-\left(\frac{L+1}{2}-M\right) B .
$$

\subsection{Singlet-triplet gap}

For the $J_{2}=0$ spin chain with open boundary conditions, the result Eq. (3.13) is modified, to first order in the marginally irrelevant bulk coupling constant, $g(L)$ to $[18]$ :

$$
\Delta_{S T}(L)=\frac{\pi v_{s}}{L}[1-g(L)]
$$

We first compute $\Delta_{S T}(L)$ in the clean case $\left(J_{K}^{\prime}=1\right)$ using the Bethe Ansatz solution up to $L=10^{4}$ to extract the marginal coupling $g(L)$ from Eq. (4.11). A very good agreement (for $L \gg 100$ ) with $g(L)$ from Eq. (2.23) is observed using $L_{1} \simeq 0.92$.

For $J_{K} \neq 1$ we expect Eq. (4.11) to continue to hold in the strong coupling limit of the SCKM, $\xi_{K} \ll L$, but we may also include the Fermi liquid correction of Eq. (3.14):

$$
\Delta_{S T}\left(L \gg \xi_{K}\right)=\frac{\pi v_{s}}{L}\left[1-g(L)-\frac{\pi \xi_{K}}{2 L}\right] .
$$

Note that this result is to first order in $g(L)$ and first order in $\xi_{K} / L$ only. It should be corrected by a Taylor series in these 2 coupling constants. We also emphasize that the $g(L) \sim 1 / \ln (L)$ term is the dominant correction in this expression. Hence, in order to verify the presence of the term proportional to $\xi_{K}$ it is natural to study the following

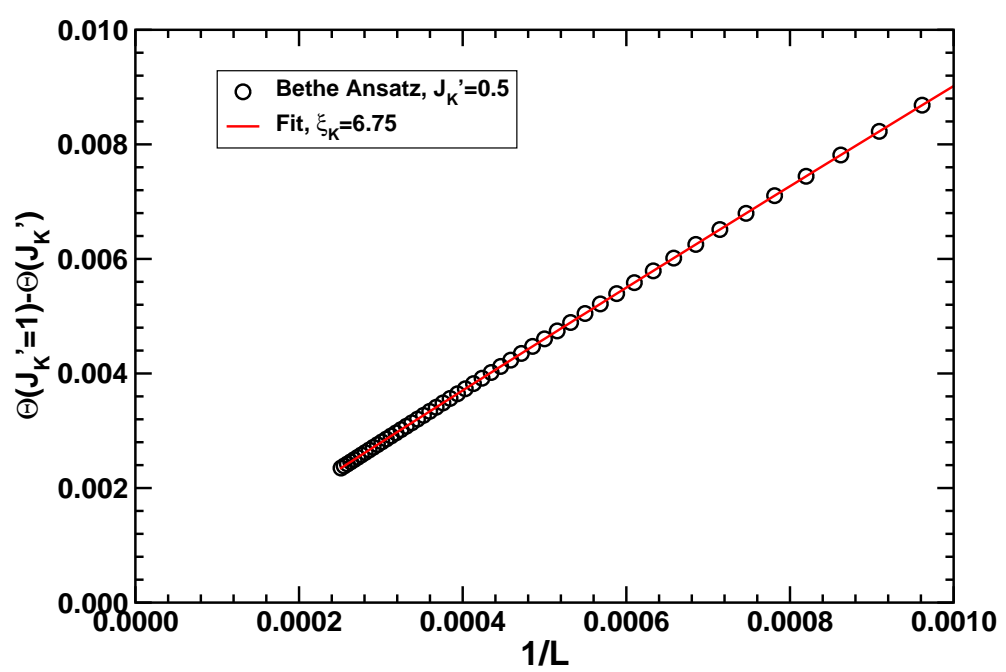

Figure 9. Bethe Ansatz results for the $\operatorname{SCKM}\left(J_{2}=0\right)$. Data are shown for the difference $\Theta\left(J_{K}^{\prime}=1\right)-\Theta\left(J_{K}^{\prime}\right)$ [Eq. (4.13)] computed exactly for system sizes $20 \leq L \leq 4000$ with an impurity coupling $J_{K}^{\prime}=0.5$. The solid line represents a fit to the data of the form Eq. (4.13) with $\xi_{K}=6.75, a=-10.21, b=-109.39$. 
quantity:

$$
\begin{aligned}
\frac{L}{\pi v_{s}} \Delta_{S T}^{\prime}\left(J_{K}^{\prime}\right) & =\frac{L}{\pi v_{s}}\left[\Delta_{S T}\left(J_{K}^{\prime}=1\right)-\Delta_{S T}\left(J_{K}^{\prime}\right)\right] \\
& \equiv \Theta\left(J_{K}^{\prime}=1\right)-\Theta\left(J_{K}^{\prime}\right) \\
& \sim \frac{\pi\left(\xi_{K}-\xi_{K}\left(J_{K}^{\prime}=1\right)\right)}{2 L}+\frac{a}{L \ln (L)}+\frac{b}{L^{2}}, \quad L \gg \xi_{K}
\end{aligned}
$$

where we have included the leading terms in the expected Taylor series in the 2 coupling constants. In particular, one might expect a term of the form $\sim\left(\pi v_{s} / L\right) g(L) \xi_{K} / L \sim$ $1 /\left(L^{2} \ln (L)\right)$ to be present in the finite-size corrections to $\Delta_{S T}(L)$. The analytical calculation of the coefficient of such a term would be challenging since it would involve diagrams with 6 current operators. In the opposite limit $L \ll \xi_{K}$ we can calculate $\Delta_{S T}(L)$ in perturbation theory in $\lambda_{K}$, Eq. (3.15). At weak coupling $\left(L \ll \xi_{K}\right)$ there is no correction of $O(g)$. This follows because both singlet and triplet states arise from the same state (with $S=1 / 2$ ) of the chain of $L$ spins.

Exact results available form the Bethe ansatz solution of finite systems allow us to verify the presence of the $\pi \xi_{K} /(2 L)$ term in the singlet-triplet gap, Eqs. (4.12), (4.13). Our results for $\Theta\left(J_{K}^{\prime}=1\right)-\Theta\left(J_{K}^{\prime}\right)$ are shown in Fig. 9 for $J_{K}^{\prime}=0.5$, clearly demonstrating the presence of a linear term $\sim\left(\xi_{K}-\xi_{K}\left(J_{K}^{\prime}=1\right)\right) / L$. If the higher order corrections described by Eq. (4.13) are included a very precise absolute estimate for $\xi_{K}-\xi_{K}\left(J_{K}^{\prime}=1\right)$ can be obtained. We expect $\xi_{K}\left(J_{K}^{\prime}=1\right)$ to be $\mathcal{O}(1)$ and therefore to be negligible compared to $\xi_{K}$ for large enough $\xi_{K}$. Fits to the form given by Eq. (4.13)

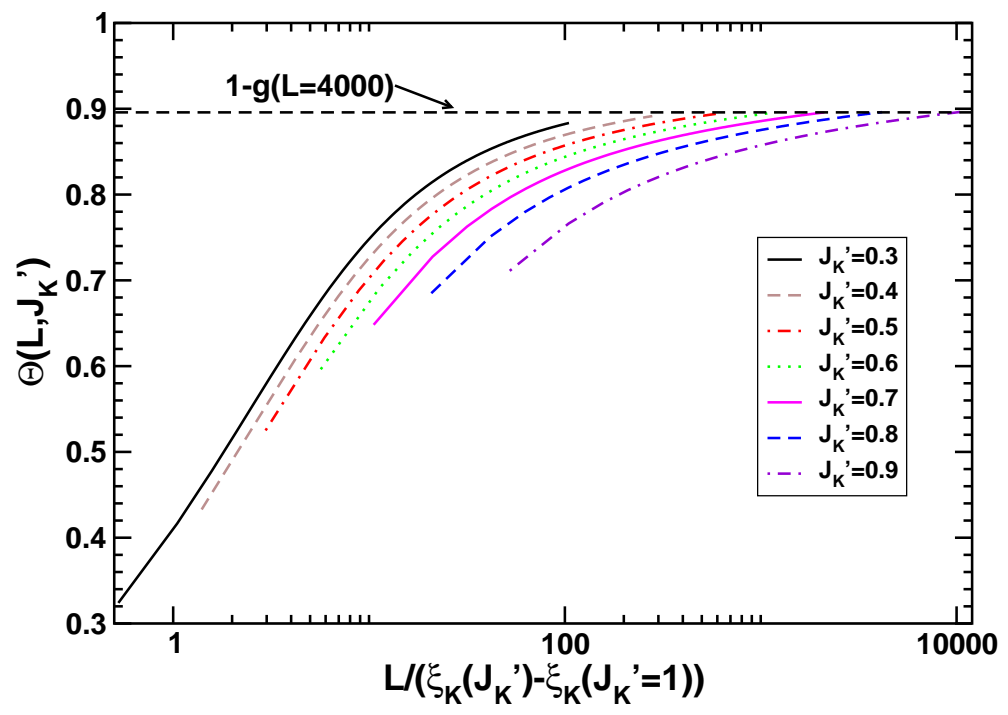

Figure 10. Bethe Ansatz results for the SCKM $\left(J_{2}=0\right)$. Data are shown for the singlet-triplet gap $\Delta_{S T}$ versus $L /\left(\xi_{K}-\xi_{K}\left(J_{K}^{\prime}=1\right)\right)$ computed exactly for various system sizes $20 \leq L \leq 4000$ and impurity couplings $J_{K}^{\prime}=0.3, \ldots, 0.9$ as indicated in the plot by the different symbols. $\xi_{K}-\xi_{K}\left(J_{K}=1\right)$ is obtained from fitting to Eq. (4.13) as listed in table 4. Due the the presence of the marginal coupling, $g$, the data clearly do not scale with $L / \xi_{K}$ as was the case at the critical point $J_{2}^{c}$ as shown in Fig. 5. 


\begin{tabular}{|r||ccccccc|}
\hline$J_{K}^{\prime}$ & 0.3 & 0.4 & 0.5 & 0.6 & 0.7 & 0.8 & 0.9 \\
\hline$\xi_{K}-\xi_{K}\left(J_{K}^{\prime}=1\right)$ & 38.12 & 14.32 & 6.75 & 3.53 & 1.90 & 0.96 & 0.38 \\
\hline
\end{tabular}

Table 4. Bethe Ansatz estimates for the Kondo length scale $\xi_{K}-\xi_{K}\left(J_{K}^{\prime}=1\right)$ of the SCKM Hamiltonian Eq. (1.2), $J_{2}=0$, with a Kondo coupling $J_{K}^{\prime}$.

are extremely good down to $J_{K}^{\prime}=0.3$ and the obtained values of $\xi_{K}-\xi_{K}\left(J_{K}^{\prime}=1\right)$ are listed in table 4 . For smaller values of $J_{K}^{\prime}$ the diverging $\xi_{K}$ prohibits reliable fits for the system sizes where we can numerically solve the Bethe ansatz equations.

As we showed in Fig. 5, the quantity $\Theta=L /\left(\pi v_{s}\right) \Delta_{S T}\left(L, J_{K}^{\prime}\right)$ scales with $L / \xi_{K}$ in the FEKM and also for the SCKM at $J_{2}^{c}$ where the bulk marginal coupling, $g$, is zero. At $J_{2}=0, g$ is non-zero and is in fact the dominant correction term in singlettriplet gap, Eq. (4.12). Hence, for $J_{2}=0$ we no longer expect scaling of $\Theta$ with $L / \xi_{K}$, but instead, $\Theta$ should approach $1-g(L)$ for large $L$. This is demonstrated in Fig. 10 where exact Bethe ansatz results for $\Theta$ for the SCKM at $J_{2}=0$ are plotted versus $L /\left(\xi_{K}-\xi_{K}\left(J_{K}^{\prime}=1\right)\right.$ with $\xi_{K}-\xi_{K}\left(J_{K}^{\prime}=1\right)$ obtained from fitting to Eq. (4.13) as listed in table 4. Clearly the data do not scale, even for sizable values of $\xi_{K}-\xi_{K}\left(J_{K}^{\prime}=1\right)$ where $\xi_{K}\left(J_{K}^{\prime}=1\right)$ can be neglected, but approach the value $1-g(L)$ plotted as the dashed line with $g(L)$ from Eq. (2.23) using the previously determined $L_{1} \simeq 0.92$. Data are shown for $20 \leq L \leq 4000$. From Eq. (4.12) it would be tempting to assume that the quantity $\Theta\left(J_{K}^{\prime}=1\right)-\Theta\left(J_{K}^{\prime}\right)$ should scale with $L / \xi_{K}$ for the SCKM at $J_{2}=0$, however, higher order terms in $g(L)$ not included in Eq. (4.12) do not cancel, corrupting the scaling. Likewise, we also do not expect $\Theta$ to scale with $L / \xi_{K}$ in the weak coupling regime $L \ll \xi_{K}$ since, even though there is no correction of $\mathcal{O}(g)$, since $\lambda_{K}(L)$, Eq. (2.9), is not a pure scaling function of $\xi_{K} / L$, unlike in the FEKM.

\subsection{Finite size scaling of the ground state energy in the SCKM with $J_{2}=0$}

In section 4.4, Eq. (3.19), we derived the finite size corrections to the ground-state energy neglecting the bulk marginal operator, $g$. We now discuss the more general case where $g>0$ and logarithmic corrections arising from this operator are present. For the uniform $\left(J_{K}^{\prime}=1\right)$ chain with open boundary conditions these corrections are known [18]. In addition, as outlined in Appendix A, it is also possible to derive the universal term in the ground-state energy arising from the coupling between $g$ and $\xi_{K}$ :

$$
\delta E_{G S}=\frac{\xi_{K} g v_{s} \pi^{2}}{32 L^{2}} .
$$

Combining the various results, we find for general $J_{2} \leq J_{2}^{c}$ and $J_{K}^{\prime} \neq 1$ with $L \gg \xi_{K}$ :

$$
\begin{aligned}
E_{\mathrm{GS}}\left(L, J_{K}^{\prime}\right)=e_{0}\left(J_{K}^{\prime}\right) & +e_{1} L-\frac{\pi v_{s}}{24 L}\left[1-\frac{9 g^{2}}{2}\right] \\
& +\left(e_{2}+\frac{\pi^{2} v_{s} \xi_{K}}{48}(1+(3 / 2) g)\right) \frac{1}{L^{2}} .
\end{aligned}
$$

Here, as $L \rightarrow \infty, g \sim 1 / \ln L$. 
We have attempted to verify the correction to the ground-state energy, Eq. (4.14), for non-zero $g$ through the use of the Bethe ansatz solution for the SCKM at $J_{2}=0$ [8]. Although a logarithmic term of the correct order can clearly be identified, the sign appears to be the opposite of Eq. (4.14). This may be due to significant higher order corrections that could change the effective sign observed in the numerical results.

\subsection{Bethe ansatz results for the magnetization, $M(H)$}

FZ gave results on the magnetization for the SCKM at zero temperature at infinite length using the Bethe Ansatz. They found [8, 22] that, at large $L$, the magnetization had the form:

$$
M(L)=m_{0} L+\left(M_{\text {edge }}^{z}+M_{i m p}^{z}\right)+O(1 / L) .
$$

Here $m_{0}$ is the bulk magnetization per unit length, which is linear in $H$ (up to logarithmic corrections) at $H \ll J$. For $H \ll J$, FZ found:

$$
M_{\text {edge }}^{z}=\frac{1}{4}\left[\frac{1}{\ln \left(H_{0} / H\right)}-\frac{1}{2} \frac{\ln \left[(1 / 2) \ln \left(H_{0} / H\right)\right]}{\ln ^{2}\left(H_{0} / H\right)}\right]+\ldots
$$

FZ wrote $M_{i m p}^{z}$ in terms of a field

$$
H_{-} \equiv H_{0} e^{-\pi\left|\tilde{\lambda}_{K}\right|}
$$

Here:

$$
H_{0} \equiv J \sqrt{\pi^{3} / e}
$$

and $\tilde{\lambda}_{K}$ is defined by:

$$
J_{K}^{\prime}=\frac{1}{1+\tilde{\lambda}_{K}^{2}} .
$$

They identified $H_{-}$with a Kondo temperature, $T_{K}$. At $J_{K}^{\prime} \ll 1$ this has the exponential square-root form derived in section 2.4 from the RG:

$$
T_{K}=H_{0} e^{-\pi \sqrt{1 / J_{K}^{\prime}-1}} .
$$

At $H \ll T_{K} \mathrm{FZ}$ found that $M_{i m p}^{z}$ vanishes (for an $\mathrm{S}=1 / 2$ impurity). However, for $T_{K} \ll H \ll J$, they found:

$$
\begin{aligned}
M_{\mathrm{imp}}^{z}(H) \rightarrow & \frac{1}{2}-\frac{1}{4}\left[\frac{1}{\ln \left(H / T_{K}\right)}+\frac{\ln \left[(1 / 2) \ln \left(H / T_{K}\right)\right]}{\ln ^{2}\left(H / T_{K}\right)}\right] \\
- & \frac{1}{4}\left[\frac{1}{\ln \left(H_{0}^{2} / H T_{K}\right)}+\frac{1}{2} \frac{\ln \left[(1 / 2) \ln \left(H_{0}^{2} / H T_{K}\right)\right]}{\ln ^{2}\left(H_{0}^{2} / H T_{K}\right)}\right], \\
& \left(T_{K} \ll H \ll J\right) .
\end{aligned}
$$

For the model with $J_{K}^{\prime}=1$, Furusaki and Hikihara [21] observed that $M_{\text {edge }}^{z}$ (which was obtained earlier by Bethe ansatz for the $J_{K}^{\prime}=1$ case in related models [23, 24]) can be derived from renormalization group improved first order perturbation theory in $g$. It is simply $(1 / 4) g(H)$, up to a higher order term (at small $H$ ) of $O\left[1 / \ln ^{2}\left(H_{0} / H\right)\right]$. We should associate terms $(1 / 8) g(H)$ with each boundary. 
It is interesting to consider the "impurity magnetization" $\mathcal{M}_{i m p}$ as it is usually defined in the theory of the Kondo effect. This is the additional magnetization resulting from adding the impurity. For the spin chain this is naturally defined as:

$$
\mathcal{M}_{\text {imp }} \equiv \lim _{L \rightarrow \infty}\left[M\left(J_{K}, L+1\right)-M\left(J_{K}=1, L\right)\right] .
$$

Ignoring a term $m_{0}(H)$, the bulk magnetization per unit length, which is smaller by a factor of $(H / J)$ than the other contributions, we see that:

$$
\mathcal{M}_{i m p}=M_{i m p}^{z} \text {. }
$$

The first line in $\mathcal{M}_{i m p}$ in Eq. (4.22) is precisely the standard result for the FEKM, at $T_{K} \ll H \ll D$, up to corrections of $O\left[1 / \ln ^{2}\left(H / T_{K}\right)\right]$. (See, for example, [2].) It is, of course, a scaling function of $H / T_{K}$. The second line in $\mathcal{M}_{i m p}$ in Eq. (4.22) is rather complicated. Using:

$$
\ln \left(H T_{K} / H_{0}^{2}\right)=2 \ln \left(H / H_{0}\right)+\ln \left(T_{K} / H\right)
$$

we can write it in terms of $g(H)$ and $\lambda_{K}(H)$.

$$
\delta M_{i m p} \approx \frac{-1 / 4}{2 / g(H)+1 / \lambda_{K}(H)} .
$$

This could be Taylor expanded in powers of $g(H)$ and indeed has the form of Eq. (2.38). However, we find the peculiar non-analytic form of Eq. (4.26) puzzling.

$$
\frac{1}{2 / g+1 / \lambda_{K}}=\frac{g}{2} \sum_{n=0}^{\infty}\left(\frac{-g}{2 \lambda_{K}}\right)^{n} .
$$

Clearly, this expression does not have a joint Taylor expansion in $g$ and $\lambda_{K}$.

\section{Conclusions}

Our main results for $\xi_{K}=v_{s} / T_{K}$ are summarized in Fig. 11 for the SCKM at $J_{2}=0$, panel (a), and $J_{2}=J_{2}^{c}$, panel (b). We have shown that the spin chain Kondo model with the second neighbor coupling, $J_{2}$ adjusted to the critical point has the same low energy, long distance behavior as the usual free electron Kondo model. This leads to the usual exponential divergence of $\xi_{K}$ with $1 / J_{K}^{\prime}$ as shown in panel (b) where the concordance of $\xi_{K}$ as obtained from scaling of $\Delta_{S T}, E_{g s}$ and RG is demonstrated. Furthermore, at $J_{2}=J_{2}^{c}$ many quantities display scaling with $L / \xi_{K}$ in an identical manner for the SCKM and FEKM (Fig. 5). For $J_{2}<J_{2}^{c}$ a new kind of Kondo effect arises in the SCKM due to the presence of the non-zero marginal coupling $g$. As shown in panel (a) for $J_{2}=0$ this leads to a more slowly diverging $\xi_{K} \sim \exp \left(\sqrt{1 / J_{K}^{\prime}-1}\right)$ in agreement with the analytical Bethe ansatz result [8]. The non-zero marginal coupling $g>0$ destroys scaling with $\xi_{K} / L$ and reliable estimates for $\xi_{K}$ can only be obtained from a numerical determination of finite-size corrections. However, the strong coupling fixed point remains that of a completely screened impurity and the usual Kondo physics is still present in this case albeit obscured by significant logarithmic corrections arising from the bulk marginal operator, $g$. Hence, we expect physical quantities to behave 


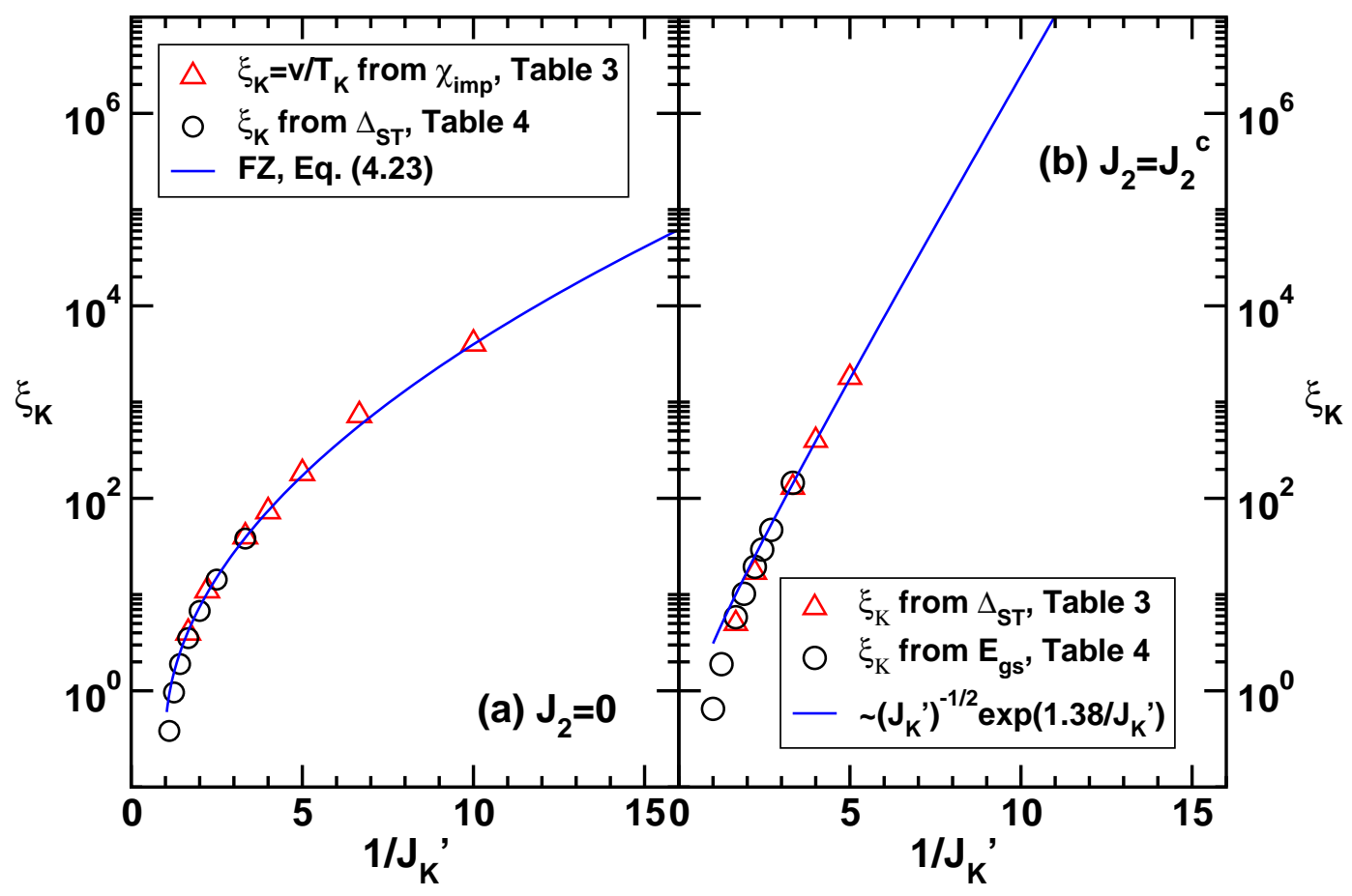

Figure 11. (a) Results for the nearest neighbor SCKM, $J_{2}=0 . \xi_{K}=v_{s} / T_{K}$ as obtained from the impurity susceptibility, $\chi_{i m p}$ (table 3) shown with $\xi_{K}$ obtained from scaling of $\Delta_{S T}$ (table 4). Both quantities are seen to agree with $v / T_{K}$ from FZ, Eq. (4.21). (b) Results for the next-nearest neighbor SCKM with $J_{2}=J_{2}^{c}$. $\xi_{K}$ are shown as obtained from scaling of $\Delta_{S T}$ and from the ground-state energy. Agreement is observed with the RG result, Eq. (2.10).

the same way as for the FEKM with corrections which only vanish logarithmically with decreasing energy or increasing system size:

$$
f\left(L, J_{K}^{\prime}\right)=f_{0}\left(L / \xi_{K}\right)+g(L) f_{1}\left(L / \xi_{K}\right)+g^{2}(L) f_{2}\left(L / \xi_{K}\right)+\ldots,
$$

where $f_{0}\left(L / \xi_{K}\right)$ is the universal scaling function occurring for the FEKM.

Several open problems pertaining to the Kondo effect as it occurs in the SCKM at $J_{2}=0(g>0)$ remain. First, as briefly mentioned in section 4.1 we expect that the bulk marginal interaction will give rise to small corrections to the susceptibility, precise analytical or numerical results for such corrections would be desirable. Secondly, in section 4.4 we derived an analytical result for the coefficient of the $\left(\pi v_{s} / L\right) g(L) \xi_{K} / L$ term in the ground-state energy, Eq. (4.14), lending support to the proposed form of the scaling corrections Eq. (2.38). However, even though our numerical solution of the Bethe ansatz equations show a logarithmic term of the correct order the sign appears to be the opposite of that of Eq. (4.14). A possible explanation for this would be that higher order are significant and change the effective sign seen in the numerics. Further analytical insight to the higher order terms or drastically improved numerical results would be valuable to resolve this questions. Thirdly, additional support for the proposed scaling corrections, Eq. (2.38), in the form of an analytical calculation of the coefficient 
of the $(\pi v / L) g(L) \xi_{K} / L$ term in $\Delta_{S T}$ would also be of considerable interest. We have so far not been able to derive this coefficient in a simple manner. As pointed out in section 4.5, we find the peculiar non-analytic form of Eq. (4.26) puzzling since it does not have a joint Taylor expansion in $g$ and $\lambda_{K}$, an issue we hope will be resolved in the near future. Finally, we hope that the spin chain Kondo models studied in this paper will help experimentalists for a better understanding of impurity effects in spin chains materials.

\section{Acknowledgments}

We are grateful to M.-S. Chang, R. Pereira for interesting discussions and H. Frahm, A. Furusaki for helpful correspondence. This research was supported by NSERC (all authors), the Swiss National Fund (NL), MaNEP (NL), CIfAR (IA), CFI (ESS), and SHARCNET (ESS). ESS gratefully acknowledge the hospitality of the Kavli Institute for Theoretical Physics in Santa Barbara, where part of this work was carried out and supported by the NSF under Grant No. PHY05-51164. Numerical simulations have been performed on the WestGrid network and the SCHARCNET facility at McMaster University.

\section{Appendix A. Derivation of $g(L) \xi_{K} / L^{2}$ term in ground state energy}

We follow closely [18], which we refer to as AQ, but note the change in notation:

$$
g_{A Q}=(\sqrt{3} / 4 \pi) g, \quad \vec{J}_{A Q}=(1 / 2 \pi) \vec{J} .
$$

First let us correct (2.21) of AQ. This was meant to be the connected part but the subscript "connected", as in (2.18) was missing. More substantially, the connected Green's function should have two other terms, missing from (2.21). The correct result, with $\vec{J}$ normalized as we do is:

$$
\begin{aligned}
\left\langle\left(\vec{J}\left(z_{1}\right) \cdot \vec{J}\left(z_{2}\right)\right) J^{a}(0) J^{b}(\tau)\right\rangle_{C} & =\frac{\delta^{a b}}{z_{1} z_{2}\left(\tau-z_{1}\right)\left(\tau-z_{2}\right)} \\
& +\frac{\delta^{a b}}{4 z_{1}^{2}\left(\tau-z_{2}\right)^{2}}+\frac{\delta^{a b}}{4 z_{2}^{2}\left(\tau-z_{1}\right)^{2}} .
\end{aligned}
$$

(We dropped the $L$ subscripts but all currents are left-moving.) These last 2 terms, missing from (2.21) of AQ, do not contribute to the $g^{2} / L$ term in $E_{G S}$ although they do lead to an additional non-universal $g^{2}$ term without the $1 / L$ factor. Therefore, they make no change in the conclusions of AQ.

Now consider including the Fermi liquid interaction as well as the bulk marginal interaction:

$$
H=H_{0}-\frac{\xi_{K}}{6} \vec{J}^{2}(0)-\frac{g}{2 \pi} \int_{0}^{L} d x \vec{J}(x) \cdot \vec{J}(-x) .
$$

For infinite $L$, standard perturbation theory to first order in $g$ and $\xi_{K}$ gives:

$$
\delta E_{G S}=-\frac{\xi_{K}}{6} \frac{g}{2 \pi} \int_{-\infty}^{\infty} d \tau \int_{0}^{\infty} d x\left\langle\vec{J}^{2}(0) \vec{J}(x, \tau) \cdot \vec{J}(-x, \tau)\right\rangle_{C} .
$$


From Eq. (A.2) setting $\tau \rightarrow 0, z_{1} \rightarrow \tau+i x, z_{2} \rightarrow \tau-i x$, and summing over $a=b$, this is:

$$
\delta E_{G S}=-\frac{\xi_{K}}{6} \frac{g}{2 \pi} \int_{-\infty}^{\infty} d \tau \int_{0}^{\infty} d x \frac{9}{2(\tau+i x)^{2}(\tau-i x)^{2}} .
$$

Note that all 3 terms in Eq. (A.2) contribute with the 2 last terms giving 1/2 the contribution of the first term. Also note that the integrand is an even function of $x$ so we can extend the region of integration to $-\infty<x<\infty$. Doing the $x$-integral gives:

$$
\delta E_{G S}=-\frac{\xi_{K}}{6} \frac{g}{2 \pi} \frac{9 \pi}{8} \int_{-\infty}^{\infty} \frac{d \tau}{|\tau|^{3}} .
$$

This integral is divergent. We cut it off, $|\tau|>\tau_{0}$, giving:

$$
\delta E_{G S}=-\frac{3 \xi_{K} g}{32 \tau_{0}^{2}}
$$

This is a non-universal term with no $L$-dependence. To get the $L$-dependent term we need the generalization of Eq. (A.2) to finite $L$. This is obtained by:

$$
\tau \pm i x \rightarrow(2 L / \pi) \sinh [(\tau \pm i x) \pi / 2 L] .
$$

the $x$ and $\tau$ integrals (with the cut off on the $\tau$ integral) can again be done exactly. Again the integrand is an even function of $x$ so we can extend it to $-L<x<L$ and then change variables to $z=e^{i \pi x / L}$. The resulting contour integral is elementary, with a double pole inside the contour giving:

$$
\delta E_{G S}=-\frac{3 \xi_{K} g \pi^{3}}{32 L^{3}} \int_{-\infty}^{\infty} d \tau \frac{\cosh (\pi \tau / L)}{|\sinh (\pi \tau / L)|^{3}} .
$$

Doing the cut-off $\tau$ integral exactly gives:

$$
\delta E_{G S}=-\frac{3 \xi_{K} g}{32\left[(L / \pi) \sinh \left(\pi \tau_{0} / L\right)\right]^{2}} .
$$

Now we Taylor expand to second order in $\tau_{0} / L$ giving:

$$
\delta E_{G S}=-\frac{3 \xi_{K} g}{32}\left[\frac{1}{\tau_{0}^{2}}-\frac{\pi^{2}}{3 L^{2}}+\ldots\right] .
$$

So the desired universal term is:

$$
\delta E_{G S}=\frac{\xi_{K} g \pi^{2}}{32 L^{2}} .
$$

Reinserting the factor of $v_{s}$ which was set to 1 gives the $\mathcal{O}\left(g / L^{2}\right)$ term in Eq. (4.15).

\section{Appendix B. Extracting the $1 / L^{2}$ Term in $E_{G S}$}

In this appendix we describe a form of Richardson extrapolation that is useful for isolating the $1 / L^{2}$ term in the ground-state energy needed to extract $\xi_{K}$ in Eq. (3.19).

We assume that the ground-state energy is of the form, Eq. (3.19):

$$
E^{(0)}(L)=A c_{-1}^{0}(L)+B c_{0}^{0}(L)+C c_{1}^{0}(L)+D c_{2}^{0}(L)+\mathcal{O}\left(L^{-3}\right),
$$


with $c_{-1}^{0}(L)=L, c_{0}^{0}(L)=1, c_{1}^{0}(L)=1 / L$ and $c_{2}^{0}(L)=1 / L^{2}$. We're interested in determining the coefficient $D$. Furthermore, we assume that $E(L)$ is known for a sequence of $L$. It is then easiest to proceed using a form Richardson extrapolation by systematically removing the $A, B, C$ terms. We eliminate the term proportional to $A$ by constructing a new series:

$$
E^{(1)}(L)=E^{(0)}(L)-E^{(0)}(L+2) \frac{c_{-1}^{0}(L)}{c_{-1}^{0}(L+2)}
$$

Naturally, $E^{(1)}$ must be of the form:

$$
E^{(1)}(L)=B c_{0}^{1}(L)+C c_{1}^{1}(L)+D c_{2}^{1}(L)+\mathcal{O}\left(L^{-2}\right),
$$

with

$$
c_{i}^{1}(L)=c_{i}^{0}(L)-c_{i}^{0}(L+2) \frac{c_{-1}^{0}(L)}{c_{-1}^{0}(L+2)}, i=0,1,2 .
$$

Now we can eliminate the term proportional to $B$ by transforming:

$$
E^{(2)}(L)=E^{(1)}(L)-E^{(1)}(L+2) \frac{c_{0}^{1}(L)}{c_{0}^{1}(L+2)},
$$

which we can write as:

$$
\begin{aligned}
& E^{(2)}(L)=C c_{1}^{2}(L)+D c_{2}^{2}\left(L^{2}\right)+\mathcal{O}\left(L^{-1}\right) \\
& c_{i}^{2}(L)=c_{i}^{1}(L)-c_{i}^{1}(L+2) \frac{c_{0}^{1}(L)}{c_{0}^{1}(L+2)}, i=1,2 .
\end{aligned}
$$

Repeating once more we obtain $E^{(3)}(L)$ of the form:

$$
\begin{aligned}
& E^{(3)}(L)=D c_{2}^{3}\left(L^{2}\right)+\mathcal{O}\left(L^{-1}\right) \\
& c_{2}^{3}(L)=c_{2}^{2}(L)-c_{2}^{2}(L+2) \frac{c_{1}^{2}(L)}{c_{1}^{2}(L+2)} .
\end{aligned}
$$

From which the constant $D$ can be extracted by plotting $E^{(3)}(L) / c_{2}^{3}(L)$. 
[1] N. Andrei, K. Furuya, and J. H. Lowenstein. Solution of the kondo problem. Rev. Mod. Phys., 55(2):331-402, Apr 1983.

[2] A. C. Hewson. The Kondo Problem to Heavy Fermions. Cambridge University Press, 2000.

[3] E. S. Sørensen, M.-S. Chang, N. Laflorencie, and I. Affleck. Quantum impurity entanglement. J. Stat. Mech., page P08003, 2007.

[4] Sebastian Eggert. Numerical evidence for multiplicative logarithmic corrections from marginal operators. Phys. Rev. B, 54(14):R9612-R9615, Oct 1996.

[5] F. D. M. Haldane. Spontaneous dimerization in the $s=\frac{1}{2}$ heisenberg antiferromagnetic chain with competing interactions. Phys. Rev. B, 25(7):4925-4928, Apr 1982.

[6] Sebastian Eggert and Ian Affleck. Magnetic impurities in half-integer-spin heisenberg antiferromagnetic chains. Phys. Rev. B, 46(17):10866-10883, Nov 1992.

[7] Stefan Rommer and Sebastian Eggert. Spin- and charge-density oscillations in spin chains and quantum wires. Phys. Rev. B, 62(7):4370-4382, Aug 2000.

[8] H. Frahm and A.A. Zvyagin. The open spin chain with impurity: an exact solution. J. Cond. Matt., 9:9939, 1997.

[9] N. Andrei. Diagonalization of the kondo hamiltonian. Phys. Rev. Lett., 45(5):379-382, Aug 1980.

[10] P. B. Wiegman. Exact solution of $s-d$ exchange model at $T=0$. JETP Lett., 31:364, 1980.

[11] N. Laflorencie, E. S. Sørensen, M.-S. Chang, and I. Affleck. Boundary effects in the critical scaling of entanglement entropy in 1d systems. Phys. Rev. Lett., 96:100603, 2006.

[12] E. S. Sørensen, M.-S. Chang, N. Laflorencie, and I. Affleck. Impurity entanglement entropy and the kondo screening cloud. J. Stat. Mech., page L01001, 2007.

[13] J. A. Chakhalian, R. F. Kiefl, R. Miller, J. Brewer, S. R. Dunsiger, G. Morris, W. A. MacFarlane, J. Sonier, S. Eggert, I. Affleck, A. Keren, and M. Verdaguer. Local magnetic susceptibility of the positive muon in the quasi-one-dimensional $\mathrm{s}=1 / 2$ antiferromagnet dichlorobis (pyridine) copper (ii). Phys. Rev. Lett., 91:027202, 2003.

[14] I. Affleck. A current algebra approach to the kondo effect. Nucl. Phys. B, 336:517, 1990.

[15] I. Affleck and A. W. W. Ludwig. The kondo effect, conformal field theory and fusion rules. Nucl. Phys. B, 352:849, 1991.

[16] I. Affleck and A. W. W. Ludwig. Critical theory of overscreened kondo fixed points. Nucl. Phys. B, 360:641, 1991.

[17] S. Lukyanov. Low energy effective hamiltonian for the xxz spin chain. Nucl. Phys B, 522:533, 1998.

[18] I. Affleck and S. Qin. Logarithmic corrections in quantum impurity problems. J. Phys. A, 32:7815, 1999.

[19] H. W. J. Blöte, John L. Cardy, and M. P. Nightingale. Conformal invariance, the central charge, and universal finite-size amplitudes at criticality. Phys. Rev. Lett., 56(7):742-745, Feb 1986.

[20] Olav F. Syljuåsen and Anders W. Sandvik. Quantum monte carlo with directed loops. Phys. Rev. E, 66(4):046701, Oct 2002.

[21] A. Furusaki and T. Hikihara. Boundary contributions to specific heat and susceptibility in the spin-(1/2) xxz chain. Phys. Rev. B, 69:094429, 2004.

[22] H. Frahm. Private communication. 2007.

[23] F.H.L. Essler. The supersymmetric t - j model with a boundary. J. Phys. A, 29:6183, 1996.

[24] H. Asakawa and M. Suzuki. Boundary susceptibilities of the hubbard model in open chains. J. Phys. A, 29:7811, 1996. 